\title{
THE ENIGMATIC YOUNG, LOW-MASS VARIABLE TWA 30*
}

\author{
Dagny L. Looper ${ }^{1,11}$, Subhanjoy Mohanty ${ }^{2}$, John J. Bochanski ${ }^{3}$, Adam J. Burgasser ${ }^{3,4,11}$, Eric E. MamajeK ${ }^{5}$,

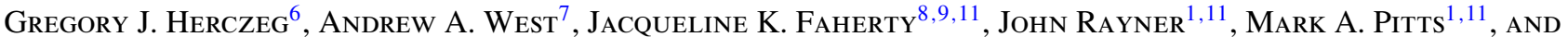 \\ J. DAVY KIRKPATRICK ${ }^{10}$ \\ ${ }^{1}$ Institute for Astronomy, University of Hawai'i, 2680 Woodlawn Drive, Honolulu, HI 96822, USA; dagny @ifa.hawaii.edu \\ ${ }^{2}$ Imperial College London, 1010 Blackett Laboratory, Prince Consort Road, London SW7 2AZ, UK \\ ${ }^{3}$ MIT Kavli Institute for Astrophysics \& Space Research, 77 Massachusetts Avenue, Building 37-664B, Cambridge, MA 02139, USA \\ ${ }^{4}$ Center for Astrophysics and Space Science, University of California San Diego, 9500 Gilman Drive, Mail Code 0424, La Jolla, CA 92093, USA \\ ${ }^{5}$ Department of Physics and Astronomy, University of Rochester, P.O. Box 270171, 500 Wilson Boulevard, Rochester, NY 14627-0171, USA \\ ${ }^{6}$ Max-Planck-Institut für extraterrestriche Physik, Giessenbachstrase, 85748 Garching, Germany \\ ${ }^{7}$ Department of Astronomy, Boston University, 725 Commonwealth Avenue, Boston, MA 02215, USA \\ ${ }^{8}$ Department of Physics and Astronomy, Stony Brook University, Stony Brook, NY 11794-3800, USA \\ ${ }^{9}$ Department of Astrophysics, American Museum of Natural History, Central Park West at 79th Street, New York, NY 10034, USA \\ ${ }^{10}$ Infrared Processing and Analysis Center, MS 100-22, California Institute of Technology, Pasadena, CA 91125, USA \\ Received 2009 October 23; accepted 2010 February 9; published 2010 April 7
}

\begin{abstract}
TWA 30 is a remarkable young $(7 \pm 3 \mathrm{Myr})$, low-mass $\left(0.12 \pm 0.04 M_{\odot}\right)$, late-type star (M5 \pm 1$)$ residing $42 \pm$ $2 \mathrm{pc}$ away from the Sun in the TW Hydrae Association (TWA). It shows strong outflow spectral signatures such as [S II], [O I], [O II], [O III], and Mg I], while exhibiting weak $\mathrm{H} \alpha$ emission (-6.8 $\pm 1.2 \AA$ ). Emission lines of [S II] and [O I] are common to T Tauri stars still residing in their natal molecular clouds, while [O III] and Mg I] emission lines are incredibly rare in this same population; in the case of TWA 30, these latter lines may arise from new outflow material colliding into older outflow fronts. The weak $\mathrm{H} \alpha$ emission and small radial velocity shifts of line emission relative to the stellar frame of rest (generally $\lesssim 10 \mathrm{~km} \mathrm{~s}^{-1}$ ) suggest that the disk is viewed close to edge-on and that the stellar axis may be inclined to the disk, similar to the AA Tau system, based on its temporal changes in emission/ absorption line strengths/profiles and variable reddening $\left(\mathrm{A}_{V}=1.5-9.0\right)$. The strong $\mathrm{Li}$ absorption $(0.61 \pm 0.13 \AA)$ and common kinematics with members of the TWA confirm its age and membership to the association. Given the properties of this system such as its proximity, low mass, remarkable outflow signatures, variability, and edge-on configuration, this system is a unique case study at a critical time in disk evolution and planet-building processes.
\end{abstract}

Key words: brown dwarfs - circumstellar matter - open clusters and associations: individual (TW Hydrae Association) - stars: evolution - stars: individual (2MASS J11321831 -3019518, TWA 30) - stars: low-mass stars: pre-main-sequence

Online-only material: color figures

\section{INTRODUCTION}

Over the past 30 years, a universal but complex paradigm of star formation has been developed through many observations and theoretical breakthroughs (e.g., Shu et al. 1987; Hartmann 1998). Briefly, the stellar birth process begins with the gravitational collapse of material within giant molecular cloud complexes (Class 0 objects; Adams et al. 1987) and progresses as the embedded protostar accretes material from an enshrouding infall envelope (Class I), with this transition lasting $\sim 200 \mathrm{kyr}$ (Enoch et al. 2008). As the gas clears, the revealed protostar continues to accrete from a circumstellar disk of material (Class II, Classical T Tauri stars, cTTS; Appenzeller \& Mundt 1989). By $\sim 10$ Myr, accretion ceases (Fedele et al. 2009; Wyatt et al. 2003), and the object is now a pre-main-sequence star, possibly with a debris disk (Class III and later) capable of assembling terrestrial planets over timescales of 10-100 Myr (Chambers 2004). This process from cloud core to pre-main-sequence star is marked by a wide array of dynamic phenomena, including outflows and accretion.

\footnotetext{
* This paper includes data gathered with the $6.5 \mathrm{~m}$ Magellan Telescopes located at Las Campanas Observatory, Chile.

${ }^{11}$ Visiting Astronomer at the Infrared Telescope Facility, which is operated by the University of Hawaii under Cooperative Agreement no. NCC 5-538 with the National Aeronautics and Space Administration, Office of Space Science, Planetary Astronomy Program.
}

Recent evidence suggests that the accretion processes at work in Class II systems operate in the regime of very low mass stars and brown dwarfs as well (e.g., White \& Basri 2003; Mohanty et al. 2005). Accretion rates scale roughly as $\dot{M} \sim M^{2}$ (Muzerolle et al. 2003; Natta et al. 2004; Calvet et al. 2004; Mohanty et al. 2005; Alexander \& Armitage 2006), meaning lower mass systems should display weaker signatures of accretion. Forbidden emission lines (FELs) in optical spectra, arising from low-density optically thin gas, have been used to identify a handful of outflows originating from very low mass stars and brown dwarfs (e.g., Fernández \& Comerón 2001; Muzerolle et al. 2003; Luhman 2004; Mohanty et al. 2005). Only five young brown dwarfs have had their outflows spatially resolved-three optical jets (via spectroastrometry; Whelan et al. 2005, 2007, 2009) and two molecular outflows (via direct imaging; Phan-Bao et al. 2008; Bourke et al. 2005). Identification of such systems is hampered not only by their intrinsic faintness but also by the distances $(d>120 \mathrm{pc})$ to the nearest star-forming regions (e.g., Sco-Cen, Taurus, Orion), making it difficult to resolve structure on the scales of disks $(\sim 10-100 \mathrm{AU})$ and jets (a few hundred AU). Hence, progress in understanding the size, morphology, and energetics of jets powered by very low mass stars and brown dwarfs is limited.

Fortunately, the recent identification of new low-mass members of the nearby TW Hydrae Association (TWA) may provide 
more ideal systems for studies of disk and jet structures in this mass regime. Indeed, one of the three brown dwarfs with a resolved optical jet, 2MASSW J1207334-393254 (2M1207AB, also known as TWA 27AB; Gizis 2002; Chauvin et al. 2004), is a member of the TWA. The namesake of the TWA, TW Hydrae, was the first cTTS found in isolation (Henize 1976)-located only $54 \pm 6$ pc from the Sun (van Leeuwen 2007), 23 degrees above the Galactic Plane and far from any molecular cloud (Herbig 1978; Rucinski \& Krautter 1983; Tachihara et al. 2009). More than a decade after the discovery of TW Hydrae, de la Reza et al. (1989) and Gregorio-Hetem et al. (1992) found four more T Tauri stars in the same vicinity by using the IRAS Point Source Catalog and targeting stars with infrared excess. Based on their common X-ray activity, Kastner et al. (1997) postulated that these five objects formed a physical association of young stars, which they termed the TWA. Soon after, targeted surveys using ROSAT All-Sky Survey (RASS) data (Sterzik et al. 1999; Webb et al. 1999; Zuckerman et al. 2001), kinematic surveys (Song et al. 2003; Scholz et al. 2005), and photometric near-infrared (NIR) surveys (Gizis 2002; Looper et al. 2007) discovered several more members, bringing the total to 23 confirmed systems (Mamajek 2005), of which five contain brown dwarfs.

These systems share similar kinematics and indicators of youth such as strong chromospheric activity and, for late$\mathrm{K}$ to M-type members, Li I $\lambda 6708$ absorption. Isochronal ages derived from the Hertzsprung-Russell diagram yield age estimates of $\sim 8 \mathrm{Myr}$ for the TWA (Soderblom et al. 1998; Webb et al. 1999; Weintraub et al. 2000; Barrado y Navascués 2006). At an average distance of 53 pc (E. E. Mamajek 2010, in preparation), the TWA is the nearest association containing actively accreting young stars, ${ }^{12}$ making it an attractive target for studying planet formation and the evolution of circumstellar disks. Moreover, members of the TWA span a range of disk evolutionary stages, with some members showing signs of actively accreting disks, passive/ non-accreting disks, or debris disks, while other members show no signs of circumstellar material (e.g., Jayawardhana et al. 1999; Low et al. 2005).

We have undertaken a survey to identify additional lowmass members to the TWA, which could provide important new case studies of accretion and outflow in substellar-mass objects residing at close distances $(\sim 50 \mathrm{pc})$. In this paper, we report the discovery of a new, low-mass member of the TWA, 2MASS J11321831-3019518, which we term TWA 30. Its membership is confirmed by its kinematics, Li I $\lambda 6708$ absorption strength, and signatures of a low-gravity photosphere. TWA 30 has strong forbidden lines of [OII, [O II], and [S II] within $30 \mathrm{~km} \mathrm{~s}^{-1}$ of the stellar rest velocity, possibly indicating an outflow from a nearly edge-on system. The presence of [O III] and $\mathrm{Mg}$ I] in the spectrum of TWA 30 is extremely rare for a cTTS and have not been observed before in a young system residing outside of its natal molecular cloud. The fluctuating continua levels from both the optical and the NIR spectra suggest that they may be affected by highly variable reddening on timescales of a day to several weeks. In Section 2, we describe the discovery and observations of TWA 30; in Section 3, we analyze the kinematics, spectral morphology, emission/absorption lines, Xray activity, and estimate an age and mass of TWA 30; in Section 4, we discuss evidence for this system having an inclined

\footnotetext{
12 Although, a recently identified young brown dwarf, 2MASS J0041353-562112, shows signs of accretion and may be a possible $\beta$ Pic or Tuc-Hor member, placing it at $\sim 35-50$ pc (Reiners 2009).
}

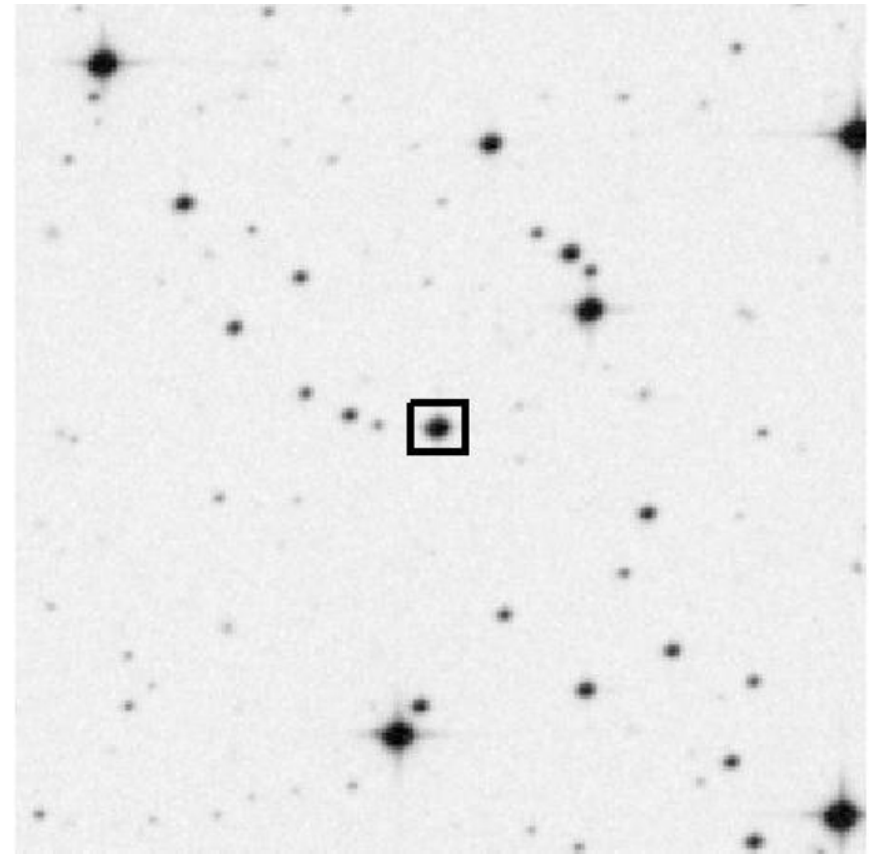

Figure 1. Finder chart for TWA 30. The field is centered on the target and is 5 arcmin on a side, with north up and east to the left. The image is in the $R$-band from the DSS II (epoch 1991 February 22 UT). A box of $20^{\prime \prime}$ on a side marks the location of the target.

stellar axis to an edge-on disk and update the disk fraction of the TWA; finally in Section 5, we give our conclusions.

\section{OBSERVATIONS}

\subsection{Discovery}

We searched for new members of the TWA that appear in the RASS X-ray Faint Source Catalog (Voges et al. 2000) over the right ascension (R.A.) range $10^{\mathrm{h}} .5-12.5$ and declination (decl.) range $-25^{\circ}$ to $-40^{\circ}$, with magnitudes of $7.5<J<$ 10 in the Two Micron All-Sky Survey (2MASS) Point Source Catalog (PSC; Cutri et al. 2003; Skrutskie et al. 2006). Previous searches of RASS data for TWA members (Webb et al. 1999; Zuckerman et al. 2001) have relied on the X-ray Bright Source Catalog (Voges et al. 1999), with the exception of the study by Sterzik et al. (1999), which examined faint sources in RASS data over a much smaller field of view than our survey. We identified TWA 30 in our sample as a faint X-ray source (1RXS J113217.7-302007) with $(2.47 \pm 0.96) \times 10^{-2}$ counts s$^{-1}, J=$ 9.64, and $J-K_{s}=0.88$. TWA 30 is located in the southwest region of the TWA (a finder chart for it is shown in Figure 1). Further details of our search methodology, spurious candidates, and other new members will be reported in a forthcoming paper.

This source was independently identified by Song et al. (2003) as SSS 113218-3019 on the basis of unpublished optical spectral data. They deduced that it is distant ( $>100 \mathrm{pc})$, and hence likely a member of the Lower Centaurus-Crux region. In the following sections, we show evidence that it is nearby and a member of the TWA.

\subsection{Optical Spectroscopic Data}

\subsubsection{MagE Spectroscopy}

TWA 30 was observed nine times with the Magellan Echellete (MagE; Marshall et al. 2008) on the $6.5 \mathrm{~m}$ Clay Telescope at 
Table 1

Spectroscopic Observation Log for TWA 30

\begin{tabular}{|c|c|c|c|c|c|c|c|}
\hline Tel./Inst. & $\lambda(\mu \mathrm{m})$ & $\lambda / \Delta \lambda$ & UT Date $^{\mathrm{a}}$ & $N \times t(\mathrm{~s})^{\mathrm{b}}$ & $Z$ & Calibrator & Conditions \\
\hline Magellan/MagE & $0.30-1.05$ & 4100 & $081126-1^{\mathrm{c}}$ & $1 \times 300$ & 1.4 & HR 3454 (WD) & Light Cirrus, 0". 5 seeing \\
\hline Magellan/MagE & $0.30-1.05$ & 4100 & $081126-2^{c}$ & $1 \times 300$ & 1.4 & HR 3454 (WD) & Light Cirrus, 0" 5 seeing \\
\hline Magellan/MagE & $0.30-1.05$ & 4100 & $081126-3^{c}$ & $1 \times 300$ & 1.4 & HR 3454 (WD) & Light Cirrus, 0'.5 seeing \\
\hline Magellan/MagE & $0.30-1.05$ & 4100 & 081127 & $1 \times 500$ & 1.5 & GD 108 (WD) & Clear, 0.'6 seeing \\
\hline Magellan/MagE & $0.30-1.05$ & 4100 & 090106 & $1 \times 500$ & 1.2 & GD 108 (WD) & Clear, $0{ }^{\prime \prime} 8$ seeing \\
\hline Magellan/MagE & $0.30-1.05$ & 4100 & 090110 & $1 \times 900$ & 1.3 & GD 108 (WD) & Clear, 0'.5 seeing \\
\hline Magellan/MagE & $0.30-1.05$ & 4100 & 090111 & $1 \times 900$ & 1.3 & GD 108 (WD) & Clear, 0 '.5 seeing \\
\hline Magellan/MagE & $0.30-1.05$ & 4100 & 090305 & $1 \times 750$ & 1.0 & GD 108 (WD) & Clear, 0.'5 seeing \\
\hline Magellan/MagE & $0.30-1.05$ & 4100 & 090531 & $1 \times 750$ & 1.1 & EG 274 (WD) & Thin Clouds, $00^{\prime \prime} 9$ seeing \\
\hline Magellan/MIKE & $0.49-0.92$ & 27000 & 081127 & $1 \times 500$ & 1.7 & HR 3454 (WD) & Clear, 0.'6 seeing \\
\hline IRTF/SpeX & $0.80-2.40$ & 1200 & 081204 & $6 \times 120$ & 1.7 & HD 92678 (A0 V) & Light Cirrus, 0'.6 seeing \\
\hline IRTF/SpeX & $1.90-4.20$ & 1500 & 081205 & $20 \times 30$ & 1.8 & HD 92678 (A0 V) & 1-2 mags Cirrus, 0".7 seeing \\
\hline IRTF/SpeX & $0.80-2.40$ & 1200 & 081215 & $12 \times 180$ & 1.7 & HD 94741 (A0 V) & Clear, $0{ }^{\prime} 8$ seeing \\
\hline IRTF/SpeX & $0.80-2.40$ & 1200 & 090202 & $10 \times 120$ & 1.6 & HD 98949 (A0 V) & Light Cirrus, 0'.5 seeing \\
\hline IRTF/SpeX & $0.80-2.40$ & 1200 & 090514 & $6 \times 100$ & 1.7 & HD 89911 (A0 V) & Clear, 0'.5 seeing \\
\hline IRTF/SpeX & $0.80-2.40$ & 1200 & 090515 & $8 \times 120$ & 1.7 & HD 110653 (A0 V) & Clear, 0'.5 seeing \\
\hline IRTF/SpeX & $0.80-2.40$ & 1200 & 090520 & $8 \times 120$ & 2.5 & HD 98949 (A0 V) & Clear, 0.8 seeing \\
\hline IRTF/SpeX & $0.80-2.40$ & 1200 & 090616 & $6 \times 180$ & 1.9 & HD 98949 (A0 V) & Light Cirrus, 0'7 seeing \\
\hline IRTF/SpeX & $0.70-2.50$ & 150 & 090628 & $6 \times 10$ & 2.1 & HD 98949 (A0 V) & Clear, 0.'5 seeing \\
\hline IRTF/SpeX & $0.70-2.50$ & 150 & 090629 & $6 \times 20$ & 1.9 & HD 98949 (A0 V) & Clear, $0{ }^{\prime} .8$ seeing \\
\hline IRTF/SpeX & $0.80-2.40$ & 1200 & 090629 & $4 \times 250$ & 2.5 & HD 98949 (A0 V) & Clear, $0{ }^{\prime} .8$ seeing \\
\hline
\end{tabular}

Notes.

a Epochs are denoted as YYMMDD in all tables.

b Number of integrations times the integration time.

c Three spectra were taken consecutively on 2008 November 26 , which we denote as 1,2 , and 3 .

Las Campanas Observatory. The details of each observation are recorded in Table 1. MagE is a medium resolution, crossdispersed echellette, covering the optical regime from 3000 to $10500 \AA$. The 0.7 slit was employed for each observation, corresponding to $R \equiv \lambda / \Delta \lambda \approx 4100$ with no binning. A flux standard was observed during each run, and ThAr arc calibration images were obtained after each science exposure. The spectrum was reduced with the MASE reduction tool pipeline (Bochanski et al. 2009). Briefly, MASE is written in the Interactive Data Language (IDL) and incorporates the entire reduction and calibration process, including bias subtraction, flat fielding, wavelength and flux calibration. Typical wavelength solutions are accurate to $\sim 5-7 \mathrm{~km} \mathrm{~s}^{-1}$. The reduced spectrum from 2009 January 10 UT is shown in Figures 2-4. Three of the spectra were taken on the same night (2008 November 26 UT) in sequential exposures and are identical to one another; we show the first spectrum of this series and the other six spectra from different epochs in Figure 5.

\subsubsection{MIKE Spectroscopy}

High-resolution spectroscopy was obtained with the Magellan Inamori Kyocera Echelle (MIKE; Bernstein et al. 2003) on 2008 November 27 UT. TWA 30 was observed with the red camera and the 0 !'7 slit, with coverage from 4900 to $9200 \AA$ and $R \sim 24,000$. Binning was set at $2 \times 2$. ThAr arcs were obtained after the science exposure, and a flux standard was observed during the night for relative flux calibration. The spectrum was reduced using the MIKE IDL pipeline ${ }^{13}$ producing a calibrated one-dimensional spectrum with wavelength solutions accurate to $\sim 1-2 \mathrm{~km} \mathrm{~s}^{-1}$.

13 The pipeline is available at http://web.mit.edu/ burles/www/MIKE/.

\subsection{NIR Spectroscopic Data}

We observed TWA 30 eleven times with the SpeX spectrograph (Rayner et al. 2003) on the $3.0 \mathrm{~m}$ NASA Infrared Telescope Facility (IRTF) in three modes with the 0.5 slit: short cross-dispersed (SXD: 0.8-2.4 $\mu \mathrm{m} ; R \sim 1200$ ), long crossdispersed (LXD: 1.9-4.2 $\mu \mathrm{m} ; R \sim 1500$ ), and prism (0.7-2.5; $R \sim 150$ ). Details of these observations are listed in Table 1 . Observations of a nearby A0 V star for telluric correction were made immediately after each observation along with internal flat-field and argon arc lamp frames for calibrations. Reductions were carried out using the Spextool package version 3.4 (Cushing et al. 2004; Vacca et al. 2003). The LXD data were first combined at each nod position; reductions using the Spextool package were otherwise standard.

\subsection{Imaging}

We observed TWA 30 at the Cerro Tololo $4.0 \mathrm{~m}$ Blanco telescope on 2008 December 13 UT as part of an ongoing brown dwarf astrometric program. The Infrared Side Port Imager (ISPI; van der Bliek et al. 2004) was used along with the $J$-band filter. The detector has a $\sim 10$ arcmin field of view with a $0^{\prime} .3$ pixel plate scale. Three images of $10 \mathrm{~s}$ exposures using three co-adds were obtained at each of the three dither positions. Dark frames and domeflats were obtained at the start of each evening. The raw images were median-combined to produce sky frames, which were then subtracted from the raw data. The subsequent reduction procedures were based on the prescriptions put together by the ISPI team, ${ }^{14}$ utilizing a combination of IRAF $^{15}$ routines as well as publicly available

\footnotetext{
14 See http://www.ctio.noao.edu/instruments/ir_instruments/ispi/.

15 IRAF is distributed by the National Optical Astronomy Observatory, which is operated by the Association of Universities for Research in Astronomy, Inc., under cooperative agreement with the National Science Foundation.
} 


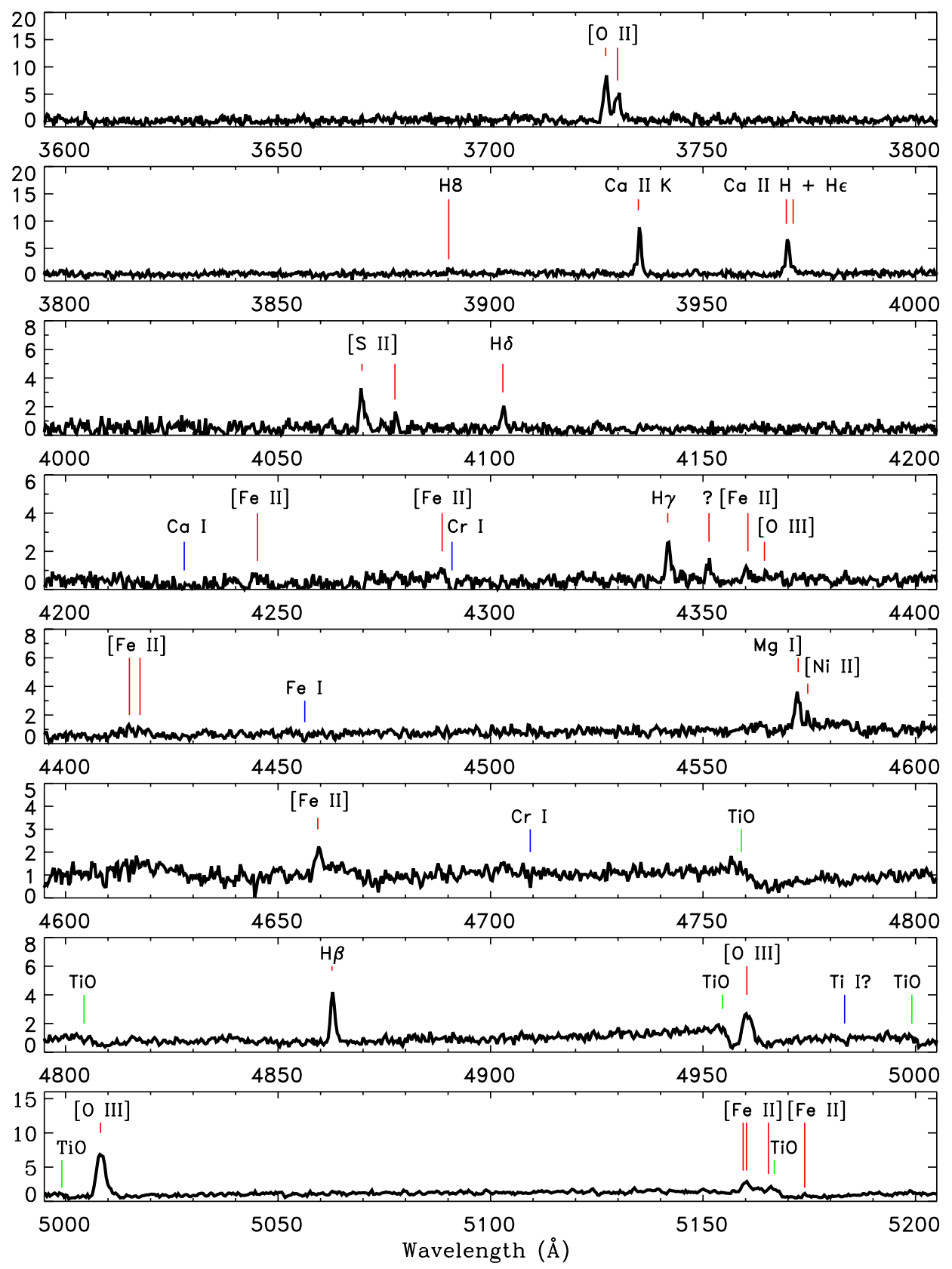

Figure 2. Optical spectrum of TWA 30, obtained with the MagE spectrograph on 2009 January 10 UT. Chromospheric emission lines and forbidden lines are indicated in red, absorption lines in blue, and molecular bands in green. Many forbidden lines typically associated with outflows (i.e., [O I], [O II], [S II]) are seen, along with strong Balmer line and Ca II emission, typical for stars with an active accretion disk. The spectral type derived from this observation is M4.

(A color version of this figure is available in the online journal.)

software packages: WCSTOOLS and SWARP. Each image was flat-fielded, corrected for bad pixels, and flipped to orient north up and east to the left using the IRAF routine osiris in the cirred package. Individual point sources were selected in each image using the IDL routine find and then input into the WCSTOOLS task imwcs, which matches stars to the 2MASS PSC. Once an initial world coordinate system (WCS) was set, we used the IRAF routine ccmap to correct for the distortion prominent across the ISPI detector. The final WCS residuals against 2MASS were $\sim 0.1$ pixels in both the $X$-and $Y$-axes. We used the software package swarp to shift and add the three reduced dither positions and then used this final science frame to perform the astrometry. Final R.A., decl. coordinate positions of $11^{\mathrm{h}} 32^{\mathrm{m}} 18^{\mathrm{s}} .19,-30^{\circ} 19^{\prime} 51^{\prime \prime}$. 8 (equinox J2000) were determined using the IRAF routine wcstran, which combines the standard WCS parameters with the higher order distortion corrections calculated with ccmap.

\section{ANALYSIS \\ 3.1. Kinematics}

TWA 30 has proper motion estimates in the USNO-B1.0 catalog (Monet et al. 2003) and the SSS catalog (Hambly et al. 2001a, 2001b, 2001c) of $\mu_{\alpha}$ and $\mu_{\delta}$ of $-86 \pm 5,-24 \pm 11$ and $-81 \pm 9,-28 \pm 9$ mas $\mathrm{yr}^{-1}$, respectively. It is also listed in the UCAC3 catalog (Finch et al. 2010) as 3UCAC 120147008 with a proper motion of $\mu_{\alpha}$ and $\mu_{\delta}$ of $-89.6 \pm 1.3$ and 

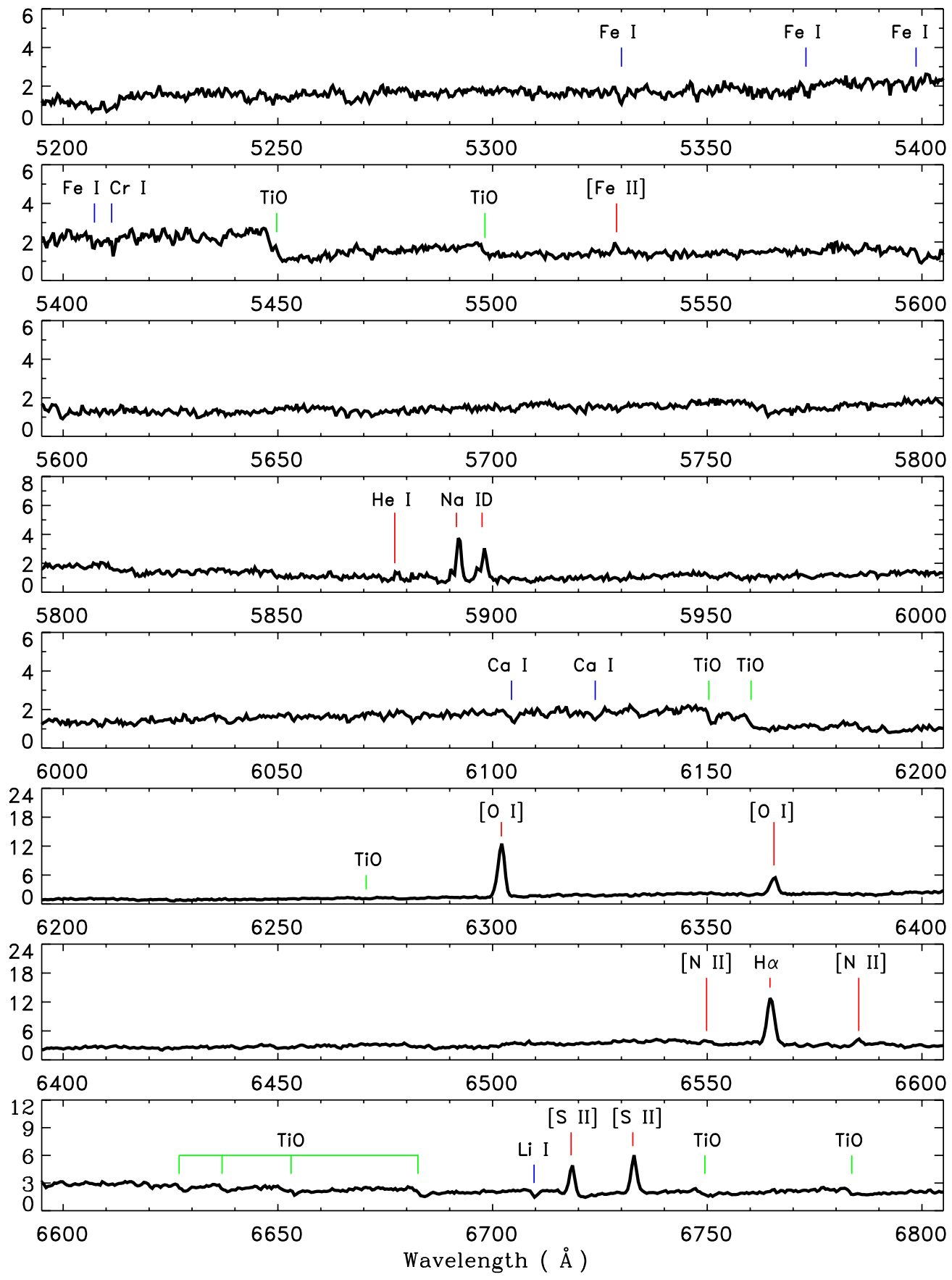

Figure 3. Same as Figure 2.

(A color version of this figure is available in the online journal.)

$-25.8 \pm 1.3$ mas $\mathrm{yr}^{-1}$. We adopt this latter measurement, which has the smallest errors, as the proper motion for TWA 30.

We determined the radial velocity (RV) of TWA 30 by measuring the line centers of $\mathrm{H} \alpha$ and photospheric absorption lines in the MIKE spectrum ( $\mathrm{Li}$ I $\lambda 6708, \mathrm{~K}_{\mathrm{I}} \lambda 7699$, and $\mathrm{Na}$ I $\lambda 8183),{ }^{16}$ yielding a heliocentric RV of $12.3 \pm 1.5 \mathrm{~km} \mathrm{~s}^{-1}$ (Table 2). The uncertainty was calculated as the $1 \sigma$ standard deviation in the measurements. To examine the RV variation over time, we calculated RVs from the nine lower resolution MagE spectra by cross-correlating each spectrum with the M6 SDSS template from Bochanski et al. (2007) using the xcorl

\footnotetext{
16 We follow the convention of referring to lines in the optical regime at their
} rest velocity in air and round to the nearest integer. package in IDL (Mohanty \& Basri 2003; West \& Basri 2009). Our analysis included the wavelength region from $6600 \AA$ to $7200 \AA$ that encompasses the TiO molecular band head that begins at $7056 \AA$. We also calculated the relative RVs by cross-correlating each MagE spectrum with the first spectrum taken chronologically on 2008 November 26 UT. These RV values indicate a relative uncertainty of $\sim 2-3 \mathrm{~km} \mathrm{~s}^{-1}$. They are consistent with each other except for the spectrum taken on 2009 March 4 UT (and possibly 2009 May 31 UT), which shows a statistically smaller RV than observed at other epochs (see Table 3).

Since no parallax measurement of TWA 30 is available, we used the convergent point analysis of Mamajek (2005) to 


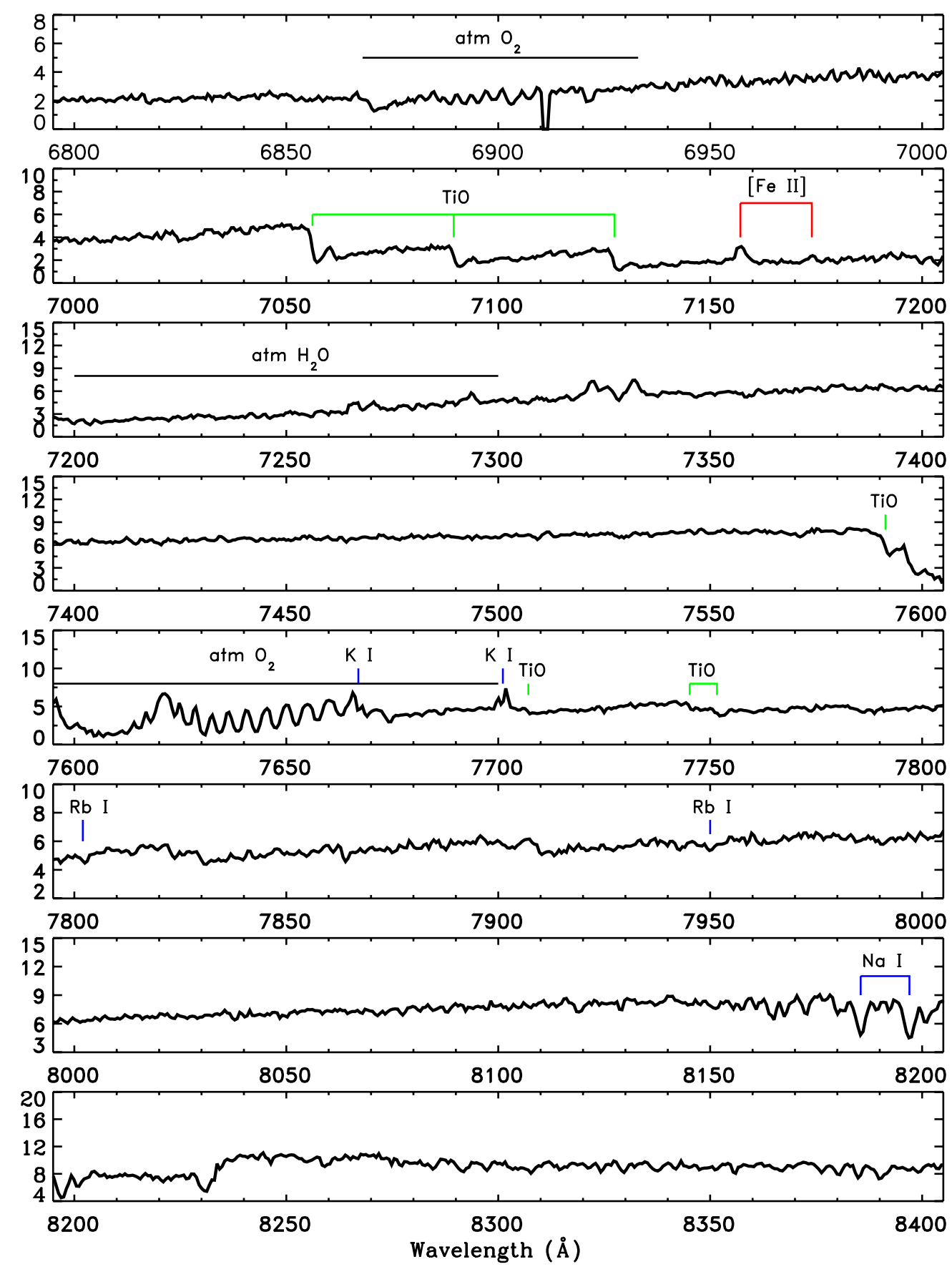

Figure 4. Same as Figure 2.

(A color version of this figure is available in the online journal.)

estimate its distance. For these calculations, we adopt the mean velocity of the TWA from Mamajek (2009) based on revised Hipparcos astrometry (van Leeuwen 2007) for TWA 1, 4, and 11 , as well as the recent ground-based astrometric analyses of 2M1207AB (TWA 27AB; Gizis et al. 2007; Biller \& Close 2007; Ducourant et al. 2008). The adopted TWA velocity is $(U, V, W)=(-10.2,-18.3,-4.9) \mathrm{km} \mathrm{s}^{-1}$, with uncertainties of $\pm 0.5 \mathrm{~km} \mathrm{~s}^{-1}$ in each component. The intrinsic one-dimensional velocity dispersion in the TWA is approximately $0.8 \mathrm{~km} \mathrm{~s}^{-1}$ (Mamajek 2005).

Most of the proper motion of TWA 30 is moving toward the TWA convergent point with near zero peculiar motion $\left(1.2 \mathrm{~km} \mathrm{~s}^{-1}\right)$, a strong indication of membership. The proper motion of TWA 30 toward the convergent point is consistent with a cluster parallax of $23.8 \pm 1.2$ mas or a distance of $42 \pm 2$ pc. This is much closer than the $>100$ pc spectrophotometric distance quoted by Song et al. (2003). Given the highly variable nature of this object (see Sections 3.2 and 3.3), the kinematic distance is more reliable. The mean distance to TWA members is 53 pc (E. E. Mamajek 2010, in preparation), with individual members having distances between $\sim 20$ and 80 pc.

We conclude that both the RV and proper motion are statistically consistent with TWA 30 being a TWA member. Using the stated distance estimate, we derive the inferred tangential velocity and the $(U, V, W)$ space motion for TWA 30 with respect to the Sun (see Table 2). For the latter, we used the procedure 
Table 2

Properties of TWA 30

\begin{tabular}{|c|c|c|}
\hline Parameter & Value & Ref \\
\hline$\alpha(\mathrm{J} 2000)^{\mathrm{a}}$ & 113218.31 & 1 \\
\hline$\delta(\mathrm{J} 2000)^{\mathrm{a}}$ & -301951.8 & 1 \\
\hline$\mu_{\alpha}$ & $-89.6 \pm 1.3 \mathrm{mas} \mathrm{yr}^{-1}$ & 2 \\
\hline$\mu_{\delta}$ & $-25.8 \pm 1.3 \mathrm{mas} \mathrm{yr}^{-1}$ & 2 \\
\hline Distance $^{\mathrm{b}}$ & $42 \pm 2 \mathrm{pc}$ & 3 \\
\hline$v_{\tan }^{\mathrm{b}}$ & $18.6 \pm 1.3 \mathrm{~km} \mathrm{~s}^{-1}$ & 3 \\
\hline$v_{\text {rad }}$ & $12.3 \pm 1.5 \mathrm{~km} \mathrm{~s}^{-1}$ & 3 \\
\hline$(U, V, W)^{\mathrm{b}}$ & $(-10.8,-19.2,-3.6) \pm(0.8,1.4,0.9) \mathrm{km} \mathrm{s}^{-1}$ & 3 \\
\hline Optical SpT & $\mathrm{M} 5 \pm 1$ & 3 \\
\hline $\operatorname{Age}^{c}$ & $7 \pm 3 \mathrm{Myr}$ & 3 \\
\hline Mass $^{c}$ & $0.12 \pm 0.04 M_{\odot}$ & 3 \\
\hline $\log L_{\mathrm{bol}} / L_{\odot}$ & $-1.64 \pm 0.09 \mathrm{dex}$ & \\
\hline$B^{\mathrm{d}}$ & 15.6 & \\
\hline$R^{\mathrm{d}}$ & 12.9 & 4 \\
\hline$I^{\mathrm{d}}$ & $11.30 \pm 0.03$ & 5 \\
\hline$J^{\mathrm{d}}$ & $9.64 \pm 0.02$ & 1 \\
\hline$H^{\mathrm{d}}$ & $9.03 \pm 0.02$ & 1 \\
\hline$K_{s}^{\mathrm{d}}$ & $8.77 \pm 0.02$ & 1 \\
\hline $\mathrm{Li} \mathrm{EW}^{\mathrm{e}}$ & $0.61 \pm 0.13 \AA$ & 3 \\
\hline $\mathrm{H} \alpha \mathrm{EW}^{\mathrm{f}}$ & $-6.8 \pm 1.2 \AA$ & \\
\hline X-ray & $(2.5 \pm 1.0) \times 10^{-2}$ counts s $^{-1}$ & 6 \\
\hline HR1 & $-0.2 \pm 0.4$ & 6 \\
\hline HR2 & $1.0 \pm 0.6$ & 6 \\
\hline $\log L_{X} / L_{\mathrm{bol}}$ & $-3.34 \mathrm{dex}$ & 3 \\
\hline
\end{tabular}

Notes.

${ }^{a}$ Coordinates are from the 2MASS Point Source Catalog at epoch 1999 March 24 UT.

${ }^{\mathrm{b}}$ Estimated, see Section 3.1.

${ }^{c}$ See Section 3.4.

${ }^{\mathrm{d}}$ Given in magnitudes. TWA 30 has time variable photometry. See references for further information.

${ }^{\mathrm{e}}$ Derived from the average equivalent widths (EWs) measured from the seven MagE spectra reported in Table 6. The stated error is the $1 \sigma$ standard deviation in the measurement.

${ }^{\mathrm{f}}$ Derived from the average EWs measured from the seven MagE spectra reported in Table 4. The stated error is the $1 \sigma$ standard deviation in the measurement.

References. (1) 2MASS (Skrutskie et al. 2006); (2) Finch et al. 2010; (3) This paper; (4) USNO-A2.0 (Monet et al. 1998); (5) DENIS (Epchtein et al. 1997); and (6) ROSAT All-Sky Survey Faint Source Catalog (Voges et al. 2000).

of Johnson \& Soderblom (1987) with updated galactic coordinate transformations from Murray (1989) and followed the convention that $U$ is positive toward the Galactic Center $(l=0$, $b=0)$.

\subsection{Optical Spectroscopy}

\subsubsection{Spectral Morphology}

We spectroscopically classified and determined the $A_{v}$ of each epoch of optical data from comparison to M3-M5.75 $\eta$ Cha templates (Luhman \& Steeghs 2004). This association has a similar age ( 7 Myr; Mamajek et al. 1999; Lawson et al. 2001) to the TWA and should therefore provide a better continuum fit with comparable surface gravities than would comparison to field templates, which have higher surface gravities. These templates are $\eta$ Cha 6 (M3), $\eta$ Cha 12 (M3.25), $\eta$ Cha 5 (M4), $\eta$ Cha 9 (M4.5), $\eta$ Cha 14 (M4.75), $\eta$ Cha 17 (M5.25), $\eta$ Cha 18 (M5.5), and $\eta$ Cha 16 (M5.75). Both template and data were normalized at $7500 \AA$, which was chosen as a normalization point due to the small amount of absorption at this wavelength (Kirkpatrick et al. 1991). Then the data were dereddened by

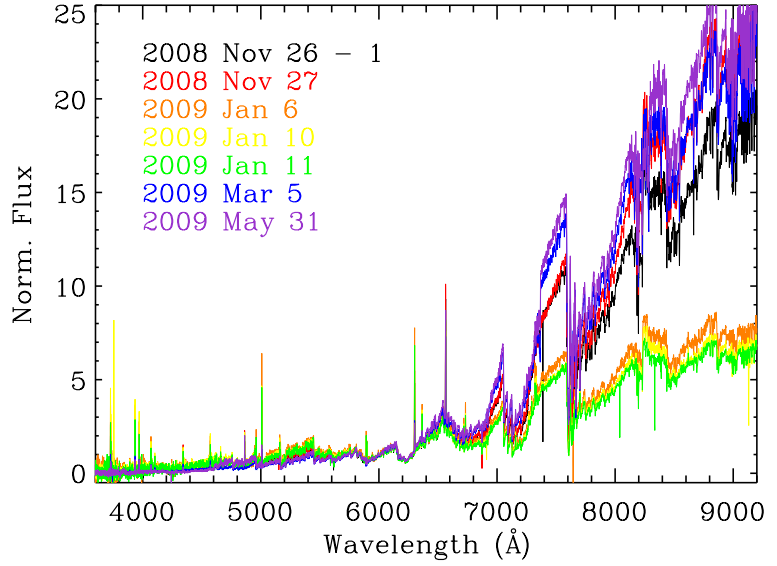

Figure 5. Optical spectra of TWA 30 taken with MagE at the seven indicated epochs. Three spectra were taken sequentially on 2008 November 26 UT, and we only show the first one here as all three are identical. The spectra have been normalized at $6000 \AA$, showing the highly variable nature of this object in both emission lines and the amount of reddening present.

(A color version of this figure is available in the online journal.)

Table 3

Radial Velocity Measurements for TWA 30 from MagE Data

\begin{tabular}{lcc}
\hline \hline UT Date & Abs. RV $\left(\mathrm{km} \mathrm{s}^{-1}\right)^{\mathrm{a}}$ & Rel. RV $\left(\mathrm{km} \mathrm{s}^{-1}\right)^{\mathrm{b}}$ \\
\hline $081126-1$ & 15.1 & N/A \\
$081126-2$ & 12.5 & -1.1 \\
$081126-3$ & 13.4 & -2.3 \\
081127 & 11.3 & -4.0 \\
090106 & 16.7 & 2.1 \\
090110 & 13.5 & -1.8 \\
090111 & 14.8 & 0.0 \\
090305 & 5.9 & -8.2 \\
090531 & 11.0 & -6.0 \\
\hline
\end{tabular}

Notes.

${ }^{a}$ These measurements were made by cross-correlation with radial velocity standards, see Section 3.1.

b These measurements were made by cross-referencing each spectrum to the 081126-1 UT spectrum and determining the relative offset.

increments of $A_{v}=0.1$ using the reddening law of Cardelli et al. (1989) (updated in the near-UV by O'Donnell 1994) to provide the best match to the template spectrum over the wavelength range $6000-8000 \AA$. We set the ratio of total-toselective extinction $R_{V} \equiv A_{V} / E(B-V)$ to 3.1 (the standard for the interstellar medium (ISM)), although large grain sizes would have higher $R_{V}$ values (e.g., Weingartner \& Draine 2001). The spectral features over this regime cover many broadband features typically used to classify $\mathrm{M}$ dwarfs such as TiO, VO, and $\mathrm{CrH}$ (Kirkpatrick et al. 1991). The best fit to the continuum, by eye, was then chosen, which simultaneously determined the spectral type and $A_{v}$.

The optimal fit of each epoch is overlaid in Figure 6. The spectral type fits of the seven epochs varied between M4 and M5.25, which span timescales of hours to weeks between epochs. The three spectra obtained on 2008 November 26 UT have a best fit to the M4.75 template, the spectrum obtained on 2008 November 27 UT has a best fit to an M4.5 template, the 2009 January 6,10 , and 11 UT spectra have a best fit to an M4 template, and the 2009 March 5 and 2009 May 31 UT have a best fit to an M5.25 template. We discuss the possible underlying cause for these changes in spectral type, M4-M5.25, in Section 4.2. 


\subsubsection{Emission and Absorption Lines}

The alkali line strengths of $\mathrm{Na}$ I and $\mathrm{K}$, which are sensitive indicators of surface gravity (Luhman 1999; Martín et al. 1999; Kirkpatrick et al. 2006; Cruz et al. 2009), are weaker in all of the optical spectra of TWA 30 than in the template dwarf spectra, indicating that this object has a lower surface gravity and is therefore younger than field dwarfs of similar spectral type. However, K I $\lambda 7699$ goes into emission in the 2009 January epochs, introducing some uncertainty in using it as a lowgravity indicator. We measured the equivalent widths (EW) of these lines along with many permitted and forbidden emission features ${ }^{17}$ over our seven epochs of MagE data and report these in Tables 4-6 and show them in Figures 2-4.

We report the average over all nights of the $\mathrm{Li}$ I absorption and the $\mathrm{H} \alpha$ emission $\mathrm{EW}$, as these are indicators of age and accretion/chromospheric activity, respectively. The prominent Balmer emission lines are a sign of youth in low-mass stars (West et al. 2008, and references therein). Furthermore, $\mathrm{Li}$ is fully depleted within $\sim 100$ Myr for stars later than spectral type mid-K (Zuckerman \& Song 2004), placing an upper limit on the age of TWA 30. It should be noted that for substellar objects with $M<0.059-0.062 M_{\odot}$, their core temperatures never become hot enough to destroy Li (Nelson et al. 1993). The Li EW of TWA 30 $(0.61 \pm 0.13 \AA)$ is comparable to other TWA M dwarf members (0.40-0.65 $\AA$ ) and stronger than $\beta$ Pic M dwarf members (Li EW < $0.4 \AA$, age 12 Myr; Zuckerman \& Song 2004).

The FELs of [O I] and [S II] identified in Figures 2 and 3 are present in the spectra of many cTTS and are signs of an outflow and accretion (Muzerolle et al. 2003). These lines are typically associated with [N II] emission, which is only weakly present at 6548 and $6583 \AA$ A. We have also identified the high ionization species [O III] and [Fe III], which typically arise in planetary nebulae or winds from the most massive stars. They are rarely seen in cTTS but are detected in the two high accretion rate cTTS: DG Tau (K5; Hartigan et al. 1995; Raga et al. 1996) and XZ Tau B (M1; White \& Ghez 2001). The emission of the high-excitation [O III] line requires extremely high temperatures and low densities, indicative of collisional shock fronts-e.g., bow shocks formed by the jet running into the surrounding ambient medium (Schwartz 1983; Hartigan 1989; Böhm 1995; Raga et al. 1996). Given the general lack of ISM gas in TWA (Tachihara et al.2009), this medium may consist of older outflow material from TWA 30, which is overtaken and shocked by the fast moving jet.

Similarly, Mg I] $\lambda 4571$ is clearly present in our spectra but is rarely seen in the spectra of cTTS. Ercolano et al. (2008) have predicted the presence of $\mathrm{Mg}$ I] arising from gas in a circumstellar disk but noted that the detection may be difficult due to the strong continuum at these wavelengths. Mg I] has previously been detected in XZ Tau B (White \& Ghez 2001) and the EXor pre-main-sequence star VY Tauri (M0; Herbig 1990), which resides in the Taurus-Auriga Cloud.

While TWA 30 has many strong spectral signatures indicating a strong outflow and hence an actively accreting circumstellar disk, it lacks any detectable He I emission at 4471, 6678, and $7065 \AA$ and has weak emission at $5876 \AA$ (see Table 5). He I emission lines are seen in the accreting M4.75-M8 Taurus members in the sample of Herczeg \& Hillenbrand (2008) and in the accreting late-M TWA members TWA 27AB (2M1207AB)

\footnotetext{
17 These lines were identified using the Atomic Line List v2.04 maintained at http://www.pa.uky.edu/ peter/atomic/ and from Kamiński et al. (2009), and references therein.
}

and TWA 28 (Herczeg et al. 2009). He I is only weakly present in the accreting late-K TWA member TWA 1 and the early-M TWA 3A (Herczeg et al. 2009). He I emission in cTTS is thought to arise both in the infalling funnel flow (broad component) and in the accretion shock region on the stellar surface (narrow component). We propose a scenario for the absence/weakness of the He I lines in our spectra in Section 4.2.

We have also examined the RV profiles of several FELs, Balmer lines, and absorption features of our MagE spectra (see Figure 7). These profiles have been corrected for the star's rest velocity $\left(12.3 \mathrm{~km} \mathrm{~s}^{-1}\right)$ and normalized to the continuum at $-250 \mathrm{~km} \mathrm{~s}^{-1}$. Of note, the $\mathrm{K}$ I $\lambda 7699$ line goes from absorption into emission for the three 2009 January epochs. A very weak P Cygni profile is seen in the profile of $\mathrm{H} \alpha$ at the 2009 March epoch. While all seven epochs of data show Na I D $\lambda 5890,5896$ in emission, the last two epochs of data, 2009 March 5 and 2009 May 31 UT, have sharp P Cygni profiles in this feature as well, indicating that we are seeing outflowing winds along the line of sight.

\subsection{NIR Spectroscopy}

\subsubsection{Spectral Morphology and Reddening}

TWA 30 was observed over several epochs spanning nearly 7 months using 0.9-2.4 $\mu \mathrm{m}$ spectroscopy. As we did not have a comprehensive set of young (low-gravity) early-to-late-M templates in the NIR, we have used field templates and carried out the same spectral typing and $A_{v}$ determination as before in the optical data analysis. In Figure 8 , we show our eight epochs of NIR data normalized in the $K$ band, which are not taken concurrently with our optical data. From these spectra, we have determined spectral types and $A_{v}$ measurements for all eight epochs (see Table 7) by dereddening our spectra to the following field templates ${ }^{18}$ - Gl 213 (M4 V), Gl 51 (M5 V), Gl 406 (M6 V), vB 8 (M7 V), vB 10 (M8 V). These spectra show a large variability in the amount of reddening present ( $\left.A_{v}=1.5-9.0\right)$, which is also correlated with the dereddened spectral types. The epoch with the least amount of reddening, 2009 February 2, has the earliest spectral type: M4.5, while the epoch with the highest amount of reddening, 2009 May 20, has the latest spectral type: M6. We outline a possible cause for the changing spectral types in both the optical (M4-M5.25) and NIR (M4.5-M6) in Section 4.2. Given the variability in spectral type in both the optical and NIR regimes, we adopt a spectral type of M5 \pm 1

We have combined the first of these epochs, 2008 December 4 UT, with our only longer wavelength (1.9-4.20 $\mu \mathrm{m})$ spectroscopic data from 2008 December 5 UT. A significant amount of reddening is observed in some of our spectra on day-timescales; however, the $K$-band $(\sim 1.9-2.4 \mu \mathrm{m})$ overlap between these two data sets is virtually identical. We compare this wider spectral energy distribution to the M5 field template, Gl 51, in Figure 9. Both spectra have been normalized in $K$ band (at $2.1 \mu \mathrm{m}$ ), showing that TWA 30 is redder than the M5 template, although the longer wavelength $L$ band is less affected and matches the template reasonably well. We have chosen an M5 for comparison, as when we dereddened this spectrum the M5 template provided the best fit in spectral slope.

\footnotetext{
18 All template spectra were obtained from http://irtfweb.ifa.hawaii.edu/ spex/IRTF_Spectral_Library/index.html. These spectra were taken with the same setup but using the 0.3 slit, instead of the $0{ }^{\prime \prime} .5$
} slit we employed for our observations. 
Table 4

Equivalent Widths of Balmer Lines for TWA 30

\begin{tabular}{|c|c|c|c|c|c|c|}
\hline UT Date & $\lambda_{\text {lab }}(\AA)$ & $\lambda_{\text {obs }}(\AA)$ & Ion & Flux $^{a}$ & $\mathrm{EW} \pm 1 \sigma(\AA)$ & $v\left(\mathrm{~km} \mathrm{~s}^{-1}\right)^{\mathrm{b}}$ \\
\hline 081126-1 & 4102.892 & 4103.028 & $\mathrm{H} \delta$ & 6.3 & $-2.7 \pm 1.2$ & 10 \\
\hline 081126-2 & 4102.892 & 4102.864 & $\mathrm{H} \delta$ & 6.3 & $-4.6 \pm 1.5$ & -2 \\
\hline 081127 & 4102.892 & 4103.095 & $\mathrm{H} \delta$ & 6.3 & $-3.8 \pm 0.6$ & 15 \\
\hline 090106 & $\ldots$ & $\ldots$ & $\mathrm{H} \delta$ & $\ldots$ & $\ldots$ & $\ldots$ \\
\hline 090110 & $\ldots$ & $\ldots$ & $\mathrm{H} \delta$ & $\ldots$ & $\ldots$ & $\ldots$ \\
\hline 090111 & $\ldots$ & $\ldots$ & $\mathrm{H} \delta$ & $\ldots$ & $\ldots$ & $\ldots$ \\
\hline 090305 & 4102.892 & 4103.013 & $\mathrm{H} \delta$ & 4.9 & $-3.1 \pm 0.4$ & 9 \\
\hline 090531 & $\ldots$ & $\ldots$ & $\mathrm{H} \delta$ & $\ldots$ & $\ldots$ & $\ldots$ \\
\hline 081126-1 & 4341.684 & 4342.017 & $\mathrm{H} \gamma$ & 10.0 & $-6.1 \pm 1.0$ & 23 \\
\hline $081126-2$ & 4341.684 & 4341.914 & $\mathrm{H} \gamma$ & 9.0 & $-5.1 \pm 1.7$ & 16 \\
\hline $081126-3$ & 4341.684 & 4342.005 & $\mathrm{H} \gamma$ & 9.6 & $-5.8 \pm 2.4$ & 22 \\
\hline 081127 & 4341.684 & 4341.933 & $\mathrm{H} \gamma$ & 9.5 & $-5.7 \pm 0.5$ & 17 \\
\hline 090106 & $\ldots$ & $\ldots$ & $\mathrm{H} \gamma$ & $\ldots$ & $\ldots$ & $\ldots$ \\
\hline 090110 & 4341.684 & 4341.855 & $\mathrm{H} \gamma$ & 9.4 & $-5.5 \pm 1.8$ & 12 \\
\hline 090111 & $\ldots$ & $\ldots$ & $\mathrm{H} \gamma$ & $\ldots$ & $\ldots$ & $\ldots$ \\
\hline 090305 & 4341.684 & 4341.796 & $\mathrm{H} \gamma$ & 6.9 & $-4.0 \pm 0.2$ & 8 \\
\hline 090531 & $\ldots$ & $\ldots$ & $\mathrm{H} \gamma$ & $\ldots$ & $\ldots$ & $\ldots$ \\
\hline 081126-1 & 4862.683 & 4862.951 & $\mathrm{H} \beta$ & 10.3 & $-6.7 \pm 0.3$ & 17 \\
\hline $081126-2$ & 4862.683 & 4862.915 & $\mathrm{H} \beta$ & 10.2 & $-6.7 \pm 0.2$ & 14 \\
\hline 081126-3 & 4862.683 & 4862.909 & $\mathrm{H} \beta$ & 12.6 & $-8.9 \pm 1.3$ & 14 \\
\hline 081127 & 4862.683 & 4862.838 & $\mathrm{H} \beta$ & 11.2 & $-7.7 \pm 0.2$ & 10 \\
\hline 090106 & 4862.683 & 4863.037 & $\mathrm{H} \beta$ & 11.5 & $-7.5 \pm 2.6$ & 22 \\
\hline 090110 & 4862.683 & 4862.863 & $\mathrm{H} \beta$ & 10.2 & $-6.6 \pm 0.7$ & 11 \\
\hline 090111 & 4862.683 & 4862.871 & $\mathrm{H} \beta$ & 9.6 & $-6.0 \pm 1.2$ & 12 \\
\hline 090305 & 4862.683 & 4862.823 & $\mathrm{H} \beta$ & 9.4 & $-5.9 \pm 0.1$ & 9 \\
\hline 090531 & 4862.683 & 4862.816 & $\mathrm{H} \beta$ & 8.4 & $-4.9 \pm 0.2$ & 8 \\
\hline 081126-1 & 6564.610 & 6564.940 & $\mathrm{H} \alpha$ & 13.3 & $-8.0 \pm 0.1$ & 15 \\
\hline $081126-2$ & 6564.610 & 6564.857 & $\mathrm{H} \alpha$ & 12.9 & $-7.6 \pm 0.1$ & 11 \\
\hline $081126-3$ & 6564.610 & 6564.799 & $\mathrm{H} \alpha$ & 11.1 & $-5.9 \pm 0.1$ & 9 \\
\hline 081127 & 6564.610 & 6564.747 & $\mathrm{H} \alpha$ & 12.9 & $-8.1 \pm 0.1$ & 6 \\
\hline 090106 & 6564.610 & 6564.811 & $\mathrm{H} \alpha$ & 12.1 & $-6.8 \pm 0.1$ & 9 \\
\hline 090110 & 6564.610 & 6564.777 & $\mathrm{H} \alpha$ & 12.1 & $-6.8 \pm 0.1$ & 8 \\
\hline 090111 & 6564.610 & 6564.854 & $\mathrm{H} \alpha$ & 13.3 & $-8.0 \pm 0.1$ & 11 \\
\hline 090305 & 6564.610 & 6564.689 & $\mathrm{H} \alpha$ & 9.5 & $-4.6 \pm 0.1$ & 4 \\
\hline 090531 & 6564.610 & 6564.647 & $\mathrm{H} \alpha$ & 10.4 & $-5.6 \pm 0.1$ & 2 \\
\hline
\end{tabular}

Notes.

a The integrated line fluxes are given in units of $10^{-16} \mathrm{erg} \mathrm{cm}^{-2} \mathrm{~s}^{-1}$ and should only be used to calculate relative line fluxes between features in the same spectrum as our data are not photometrically calibrated and hence do not account for slit losses or non-photometric conditions.

$\mathrm{b}$ These velocities are not corrected to the stellar rest frame $\left(12.3 \pm 1.5 \mathrm{~km} \mathrm{~s}^{-1}\right)$.

We have also measured spectrophotometry on these data to determine 2MASS $J H K_{s}$ colors, as well as on our two lower resolution NIR spectra from 2009 June 28 and 29 UT, and report these in Table 7 along with the 2MASS PSC measurements from 1999 March 24 UT. The epoch of our data with the least amount of reddening, 2009 February $2\left(A_{v}=1.5\right)$, has $J H K_{S}$ magnitudes very similar to those from the 2MASS PSC ( $\Delta=0.01-0.15 \mathrm{mag}$ ) epoch. While these spectra have been flux calibrated with nearby A0 V stars, they have not been absolutely flux calibrated with photometry, and therefore do not take into account slit losses. Rayner et al. (2009) estimate that in photometric conditions absolute spectrophotometry performed on SpeX spectra calibrated with nearby A0 V stars are accurate to $10 \%$, while relative spectrophotometry are accurate to a few percent.

The colors calculated from these measurements appear to be accurate between our SXD and prism spectra (Table 7), with identical $H-K_{s}$ colors and similar $J-H(\Delta=0.06 \mathrm{mag})$ colors. These values are shown in $J H K_{s}$ color space in Figure 10. Only the 2MASS PSC colors of TWA 30 lie along the dwarf track. All other epochs lie along the reddening vector described by
Cardelli et al. (1989). The sparse coverage of our data shows a full cycle over a 6 month period, however, the rapid increase in $A_{v}$ over the period 2009 May 14-20 may indicate that the cycles are much shorter if this behavior is periodic.

\subsubsection{Emission and Absorption Lines}

As seen in the optical data, typical surface gravity indicators such as the alkali metal lines, KI and Na I, are weaker in TWA 30 than in the field M5 template (see Figures 9 and 11). While $\mathrm{H}$ Balmer emission in the optical spectrum of TWA 30 is prevalent, $\mathrm{H}$ Paschen $(\mathrm{Pa})$ is in absorption in the NIR, with the exception of $\mathrm{Pa} \gamma$, which is seen in emission in our lower resolution SXD data (see Figure 9). A typical indicator of ongoing accretion, $\mathrm{H}$ Brackett $\gamma$ can be seen only weakly in emission at $2.166 \mu \mathrm{m}$ in the $K$-band spectrum of the 2008 December 4 UT data and is absent in the 2008 December 15 and 2009 February 2 UT data (see Figure 11).

\subsection{Estimated Mass and Age}

To derive the stellar mass and age, we have used two estimates of the spectral type: (1) using the 2MASS colors, which imply 
Table 5

Equivalent Widths of Selected Non-Balmer Emission Lines for TWA 30

\begin{tabular}{|c|c|c|c|c|c|c|}
\hline UT Date & $\lambda_{\text {lab }}(\AA)$ & $\lambda_{\text {obs }}(\AA)$ & Ion & Flux $^{a}$ & $\mathrm{EW} \pm 1 \sigma(\AA)^{\mathrm{b}}$ & $v\left(\mathrm{~km} \mathrm{~s}^{-1}\right)^{\mathrm{c}}$ \\
\hline 081126-1 & 3727.090 & 3727.06 & {$[\mathrm{O} \mathrm{II}]^{\mathrm{d}}$} & 11.5 & -8.5 & -2 \\
\hline $081126-2$ & 3727.090 & 3727.05 & {$[\mathrm{O} \mathrm{II}]^{\mathrm{d}}$} & 7.8 & -37.5 & -3 \\
\hline $081126-3$ & 3727.090 & 3727.02 & {$\left[\mathrm{O}_{\mathrm{II}}\right]^{\mathrm{d}}$} & 7.1 & -24.9 & -6 \\
\hline 081127 & 3727.090 & 3726.94 & {$[\mathrm{O} \mathrm{II}]^{\mathrm{d}}$} & 10.8 & -15.6 & -12 \\
\hline 090106 & 3727.090 & 3727.29 & {$[\mathrm{O} \mathrm{II}]^{\mathrm{d}}$} & 4.2 & -17.1 & 16 \\
\hline 090110 & 3727.090 & 3727.08 & {$[\mathrm{O} \text { II }]^{\mathrm{d}}$} & 7.4 & -15.7 & -1 \\
\hline 090111 & 3727.090 & 3727.05 & {$[\mathrm{O} I \mathrm{II}]^{\mathrm{d}}$} & 4.1 & -58.9 & -3 \\
\hline 090305 & 3727.090 & 3727.09 & {$[\mathrm{O} \mathrm{II}]^{\mathrm{d}}$} & 10.2 & -14.1 & 0 \\
\hline 090531 & 3727.090 & 3727.10 & {$[\mathrm{O} I I]^{\mathrm{d}}$} & 3.7 & -30.2 & 1 \\
\hline 081126-1 & 3729.880 & 3729.91 & {$[\mathrm{O} \mathrm{II}]^{\mathrm{d}}$} & 6.2 & -5.1 & 2 \\
\hline 081126-2 & 3729.880 & 3730.08 & {$[\mathrm{O} \mathrm{II}]^{\mathrm{d}}$} & 4.9 & -14.5 & 16 \\
\hline $081126-3$ & 3729.880 & 3729.88 & {$[\mathrm{O} I \mathrm{II}]^{\mathrm{d}}$} & 2.8 & -11.7 & 0 \\
\hline 081127 & 3729.880 & 3729.92 & {$[\mathrm{O} \text { II }]^{\mathrm{d}}$} & 6.7 & -9.8 & 3 \\
\hline 090106 & 3729.880 & 3729.83 & {$[\mathrm{O} \mathrm{II}]^{\mathrm{d}}$} & 2.4 & -7.5 & -4 \\
\hline 090110 & 3729.880 & 3729.91 & {$[\mathrm{O} \mathrm{II}]^{\mathrm{d}}$} & 4.6 & -6.8 & 2 \\
\hline 090111 & 3729.880 & 3729.65 & {$[\mathrm{O} \text { II }]^{\mathrm{d}}$} & 2.4 & -14.8 & -19 \\
\hline 090305 & 3729.880 & 3729.92 & {$[\mathrm{O} I \mathrm{II}]^{\mathrm{d}}$} & 6.0 & -9.7 & 3 \\
\hline 090531 & 3729.880 & 3729.88 & {$[\mathrm{O} \mathrm{II}]^{\mathrm{d}}$} & 3.5 & -26.5 & 0 \\
\hline 081126-1 & 3934.777 & 3935.235 & $\mathrm{Ca}$ II $\mathrm{k}^{\mathrm{e}}$ & 10.2 & -68.2 & 35 \\
\hline$\ldots$ & 3934.777 & 3933.775 & CaII $\mathrm{k}^{\mathrm{e}}$ & $\ldots$ & $\ldots$ & -76 \\
\hline 081126-2 & 3934.777 & 3935.271 & Ca II $k^{\mathrm{e}}$ & 34.0 & $-29.7 \pm 6.9$ & 38 \\
\hline$\ldots$ & 3934.777 & 3933.509 & $\mathrm{CaII}^{\mathrm{e}}$ & $\ldots$ & $\ldots$ & -97 \\
\hline $081126-3$ & 3934.777 & 3935.201 & CaII $\mathrm{k}^{\mathrm{e}}$ & 5.5 & -17.0 & 32 \\
\hline$\ldots$ & 3934.777 & $\ldots$ & Ca II $\mathrm{k}^{\mathrm{e}}$ & $\ldots$ & $\ldots$ & $\ldots$ \\
\hline 081127 & 3934.777 & 3935.172 & CaII $\mathrm{k}^{\mathrm{e}}$ & 32.7 & $-28.4 \pm 1.5$ & 30 \\
\hline$\ldots$ & 3934.777 & 3933.389 & Ca II $\mathrm{k}^{\mathrm{e}}$ & $\ldots$ & $\ldots$ & -106 \\
\hline 090106 & 3934.777 & 3935.14 & $\mathrm{Ca} I I \mathrm{k}^{\mathrm{e}}$ & 4.0 & -50.7 & 34 \\
\hline$\ldots$ & 3934.777 & 3934.143 & Ca II $\mathrm{k}^{\mathrm{e}}$ & $\ldots$ & $\ldots$ & -48 \\
\hline 090110 & 3934.777 & 3935.103 & CaII $\mathrm{k}^{\mathrm{e}}$ & 7.2 & -30.9 & 25 \\
\hline$\ldots$ & 3934.777 & 3933.953 & CaII $\mathrm{k}^{\mathrm{e}}$ & $\ldots$ & $\ldots$ & -63 \\
\hline 090111 & 3934.777 & 3935.02 & Ca II k $\mathrm{k}^{\mathrm{e}}$ & $\ldots$ & -17.8 & 19 \\
\hline$\ldots$ & 3934.777 & 3933.799 & Ca II $\mathrm{k}^{\mathrm{e}}$ & $\ldots$ & $\ldots$ & -75 \\
\hline 090305 & 3934.777 & 3935.204 & Ca II $\mathrm{k}^{\mathrm{e}}$ & 34.8 & $-30.2 \pm 1.5$ & 33 \\
\hline$\ldots$ & 3934.777 & 3933.886 & Ca II $\mathrm{k}^{\mathrm{e}}$ & $\ldots$ & $\ldots$ & -9 \\
\hline 090531 & 3934.777 & 3935.09 & CaII $k^{\mathrm{e}}$ & 4.4 & -17.0 & 24 \\
\hline 081126-1 & 3969.591 & 3969.916 & $\mathrm{Ca}$ II $\mathrm{h}+\mathrm{H} \epsilon^{\mathrm{d}}$ & 17.3 & $-12.2 \pm 2.3$ & 25 \\
\hline $081126-2$ & 3969.591 & 3969.71 & $\mathrm{Ca}$ II $\mathrm{h}+\mathrm{H} \epsilon^{\mathrm{d}}$ & $\ldots$ & -6.1 & 26 \\
\hline $081126-3$ & 3969.591 & 3970.100 & $\mathrm{Ca} I \mathrm{~h}+\mathrm{H} \epsilon^{\mathrm{d}}$ & 21.7 & $-15.5 \pm 7.0$ & 38 \\
\hline 081127 & 3969.591 & 3970.044 & $\mathrm{Ca}$ II $\mathrm{h}+\mathrm{H} \epsilon^{\mathrm{d}}$ & 28.4 & $-23.3 \pm 2.8$ & 34 \\
\hline 090106 & 3969.591 & 3969.946 & $\mathrm{Ca}$ II $\mathrm{h}+\mathrm{H} \epsilon^{\mathrm{d}}$ & 26.6 & $-21.8 \pm 2.7$ & 27 \\
\hline 090110 & 3969.591 & 3969.907 & $\mathrm{Ca}$ II $\mathrm{h}+\mathrm{H} \epsilon^{\mathrm{d}}$ & $\ldots$ & -32.0 & 24 \\
\hline 090111 & 3969.591 & 3969.99 & $\mathrm{Ca} I \mathrm{~h}+\mathrm{H} \epsilon^{\mathrm{d}}$ & $\ldots$ & -84.6 & 30 \\
\hline 090305 & 3969.591 & 3969.973 & $\mathrm{Ca}$ II $\mathrm{h}+\mathrm{H} \epsilon^{\mathrm{d}}$ & 29.0 & $-24.0 \pm 2.0$ & 29 \\
\hline 090531 & 3969.591 & 3969.85 & $\mathrm{Ca} I \mathrm{~h}+\mathrm{H} \epsilon^{\mathrm{d}}$ & $\ldots$ & -33.0 & 29 \\
\hline 081126-1 & 4069.749 & 4069.918 & {$[\mathrm{~S}$ II $]$} & 19.3 & $-15.4 \pm 2.0$ & 13 \\
\hline 081126-2 & 4069.749 & 4069.829 & {$[\mathrm{~S}$ II $]$} & 8.40 & $-5.0 \pm 0.5$ & 6 \\
\hline $081126-3$ & 4069.749 & $\ldots$ & [S II $]$ & $\ldots$ & $\ldots$ & $\ldots$ \\
\hline 081127 & 4069.749 & 4069.858 & {$[\mathrm{~S}$ II $]$} & 13.5 & $-10.1 \pm 0.6$ & 8 \\
\hline 090106 & 4069.740 & 4069.68 & {$[\mathrm{~S}$ II $]$} & 2.3 & -15.4 & -4 \\
\hline 090110 & 4069.749 & 4069.666 & [S II] & 11.8 & $-7.9 \pm 1.6$ & -6 \\
\hline 090111 & 4069.749 & $\ldots$ & {$[\mathrm{S}$ II $]$} & $\ldots$ & $\ldots$ & $\ldots$ \\
\hline 090305 & 4069.749 & 4069.802 & {$[\mathrm{~S}$ II $]$} & 9.5 & $-6.6 \pm 0.3$ & 4 \\
\hline 090531 & 4069.749 & 4069.837 & {$[\mathrm{~S} \mathrm{II}]$} & 8.9 & $-6.3 \pm 2.6$ & 7 \\
\hline 081126-1 & 4077.500 & $\ldots$ & {$[\mathrm{S}$ II $]$} & $\ldots$ & $\ldots$ & $\ldots$ \\
\hline $081126-2$ & 4077.500 & $\ldots$ & {$[\mathrm{S} \mathrm{II}]$} & $\ldots$ & $\ldots$ & $\ldots$ \\
\hline 081126-3 & 4077.500 & $\ldots$ & {$[\mathrm{S}$ II $]$} & $\ldots$ & $\ldots$ & $\ldots$ \\
\hline 081127 & 4077.500 & 4077.448 & {$[\mathrm{~S} \mathrm{II}]$} & 5.2 & $-2.5 \pm 0.4$ & -4 \\
\hline 090106 & 4077.500 & 4077.57 & [S II] & $<0.6$ & $<-2.7$ & 5 \\
\hline 090110 & 4077.500 & $\ldots$ & [S II $]$ & $\ldots$ & $\ldots$ & $\ldots$ \\
\hline 090111 & 4077.500 & $\ldots$ & [S II] & $\ldots$ & $\ldots$ & $\ldots$ \\
\hline 090305 & 4077.500 & 4077.558 & {$[\mathrm{~S}$ II $]$} & 5.3 & $-2.6 \pm 0.3$ & 4 \\
\hline 090531 & 4077.500 & $\ldots$ & {$[\mathrm{S} \mathrm{II}]$} & $\ldots$ & $\ldots$ & $\ldots$ \\
\hline 081126-1 & 4364.440 & 4364.966 & [O III] & 5.4 & $-1.2 \pm 0.6$ & 36 \\
\hline $081126-2$ & 4364.440 & $\ldots$ & [O III] & $\ldots$ & $\ldots$ & $\ldots$ \\
\hline
\end{tabular}


Table 5

(Continued)

\begin{tabular}{|c|c|c|c|c|c|c|}
\hline UT Date & $\lambda_{\text {lab }}(\AA)$ & $\lambda_{\text {obs }}(\AA)$ & Ion & Flux $^{a}$ & $\mathrm{EW} \pm 1 \sigma(\AA)^{\mathrm{b}}$ & $v\left(\mathrm{~km} \mathrm{~s}^{-1}\right)^{\mathrm{c}}$ \\
\hline $081126-3$ & 4364.440 & & [O III] & & & \\
\hline 081127 & 4364.440 & 4364.786 & [O III] & 6.2 & $-2.1 \pm 0.4$ & 24 \\
\hline 090106 & 4364.440 & $\ldots$ & [O III] & $\ldots$ & $\ldots$ & $\ldots$ \\
\hline 090110 & 4364.440 & $\ldots$ & [O III] & $\ldots$ & $\ldots$ & $\ldots$ \\
\hline 090111 & 4364.440 & $\ldots$ & [O III] & $\ldots$ & $\ldots$ & $\ldots$ \\
\hline 090305 & 4364.440 & 4364.770 & [O III] & 5.3 & $-1.1 \pm 0.2$ & 23 \\
\hline 090531 & 4364.440 & $\ldots$ & [O III $]$ & $\ldots$ & $\ldots$ & $\ldots$ \\
\hline $081126-1$ & 4572.377 & 4572.388 & $\operatorname{Mg} I]$ & 7.0 & $-2.3 \pm 0.4$ & 1 \\
\hline $081126-2$ & 4572.377 & 4572.490 & $\left.\operatorname{Mg}_{I}\right]$ & 9.0 & $-4.2 \pm 0.4$ & 7 \\
\hline $081126-3$ & 4572.377 & 4572.481 & $\mathrm{Mg} \mathrm{I}]$ & 12.7 & $-7.9 \pm 1.0$ & 7 \\
\hline 081127 & 4572.377 & 4572.372 & $\left.\mathrm{Mg}_{\mathrm{I}}\right]$ & 8.6 & $-3.9 \pm 0.2$ & 0 \\
\hline 090106 & 4572.377 & 4572.310 & $\left.\mathrm{Mg}_{\mathrm{I}}\right]$ & 7.4 & $-2.9 \pm 0.9$ & -4 \\
\hline 090110 & 4572.377 & 4572.206 & $\left.\mathrm{Mg}_{\mathrm{I}}\right]$ & 7.6 & $-3.0 \pm 0.4$ & -11 \\
\hline 090111 & 4572.377 & 4572.235 & $\operatorname{Mg} I]$ & 7.6 & $-2.9 \pm 1.0$ & -9 \\
\hline 090305 & 4572.377 & 4572.365 & $\left.\mathrm{Mg}_{\mathrm{I}}\right]$ & 7.5 & $-2.8 \pm 0.1$ & -1 \\
\hline 090531 & 4572.377 & 4572.276 & $\left.\mathrm{Mg}_{\mathrm{I}}\right]$ & 6.3 & $-1.6 \pm 0.6$ & -7 \\
\hline 081126-1 & 4659.350 & 4659.183 & [Fe III] & 5.1 & $-1.7 \pm 0.2$ & -11 \\
\hline 081126-2 & 4659.350 & 4659.253 & {$[\mathrm{Fe} \mathrm{III}]$} & 5.1 & $-1.3 \pm 0.2$ & -6 \\
\hline $081126-3$ & 4659.350 & $\ldots$ & [Fe III] & $\ldots$ & $\ldots$ & $\ldots$ \\
\hline 081127 & 4659.350 & 4659.500 & {$[\mathrm{Fe} \mathrm{III}]$} & 5.3 & $-1.9 \pm 0.1$ & 10 \\
\hline 090106 & 4659.350 & $\ldots$ & [Fe III] & $\ldots$ & $\ldots$ & $\ldots$ \\
\hline 090110 & 4659.350 & 4659.590 & [Fe III] & 5.4 & $-1.7 \pm 0.3$ & 15 \\
\hline 090111 & 4659.350 & $\ldots$ & {$[\mathrm{Fe}$ III] } & $\ldots$ & $\ldots$ & $\ldots$ \\
\hline 090305 & 4659.350 & 4659.684 & [Fe III] & 5.0 & $-1.2 \pm 0.1$ & 21 \\
\hline 090531 & 4659.350 & $\ldots$ & {$[\mathrm{Fe}$ III $]$} & $\ldots$ & $\ldots$ & $\ldots$ \\
\hline $081126-1$ & 4756.020 & 4756.065 & [Fe III] & 2.3 & $-0.2 \pm 0.1$ & +3 \\
\hline $081126-2$ & 4756.020 & $\ldots$ & [Fe III] & $\ldots$ & $\ldots$ & $\ldots$ \\
\hline $081126-3$ & 4756.020 & $\ldots$ & {$[\mathrm{Fe}$ III] } & $\ldots$ & $\ldots$ & $\ldots$ \\
\hline 081127 & 4756.020 & 4756.789 & [Fe III] & 3.0 & $-0.5 \pm 0.1$ & 48 \\
\hline 090106 & 4756.020 & $\ldots$ & {$[\mathrm{Fe} \mathrm{III}]$} & $\ldots$ & $\ldots$ & $\ldots$ \\
\hline 090110 & 4756.020 & $\ldots$ & {$[\mathrm{Fe}$ III] } & $\ldots$ & $\ldots$ & $\ldots$ \\
\hline 090111 & 4756.020 & $\ldots$ & [Fe III] & $\ldots$ & $\ldots$ & $\ldots$ \\
\hline 090305 & 4756.020 & 4756.644 & [Fe III] & 2.5 & $-0.4 \pm 0.1$ & 39 \\
\hline 090531 & 4756.020 & $\ldots$ & [Fe III] & $\ldots$ & $\ldots$ & $\ldots$ \\
\hline $081126-1$ & 4960.300 & 4960.380 & [O III] & 13.2 & $-7.8 \pm 0.2$ & 5 \\
\hline 081126-2 & 4960.300 & 4960.508 & [O III] & 15.1 & $-9.4 \pm 0.2$ & 13 \\
\hline $081126-3$ & 4960.300 & 4960.452 & [O III] & 16.3 & $-10.4 \pm 0.7$ & 9 \\
\hline 081127 & 4960.300 & 4960.411 & [O III] & 12.7 & $-7.2 \pm 0.1$ & 7 \\
\hline 090106 & 4960.300 & 4960.389 & [O III] & 17.8 & $-12.2 \pm 1.8$ & 5 \\
\hline 090110 & 4960.300 & 4960.439 & [O III] & 17.9 & $-12.4 \pm 1.0$ & 8 \\
\hline 090111 & 4960.300 & 4960.303 & [O III] & 20.7 & $-15.2 \pm 3.7$ & 0 \\
\hline 090305 & 4960.300 & 4960.470 & [O III] & 10.0 & $-4.1 \pm 0.1$ & 10 \\
\hline 090531 & 4960.300 & 4960.570 & [O III] & 9.0 & $-3.1 \pm 0.4$ & 16 \\
\hline $081126-1$ & 5008.240 & 5008.261 & [O III] & 33.6 & $-25.1 \pm 0.2$ & 1 \\
\hline $081126-2$ & 5008.240 & 5008.277 & [O III] & 31.6 & $-23.1 \pm 0.3$ & 2 \\
\hline $081126-3$ & 5008.240 & 5008.228 & [O III] & 36.4 & $-27.9 \pm 0.6$ & -1 \\
\hline 081127 & 5008.240 & 5008.219 & [O III] & 33.3 & $-24.8 \pm 0.3$ & -1 \\
\hline 090106 & 5008.240 & 5008.78 & [O III] & $\ldots$ & -49.2 & 32 \\
\hline 090110 & 5008.240 & 5008.337 & [O III] & 34.2 & $-25.6 \pm 0.7$ & 6 \\
\hline 090111 & 5008.240 & 5008.313 & [O III] & 32.9 & $-24.6 \pm 1.0$ & 4 \\
\hline 090305 & 5008.240 & 5008.268 & [O III $]$ & 18.9 & $-10.5 \pm 0.1$ & 2 \\
\hline 090531 & 5008.240 & 5008.299 & [O III] & 18.0 & $-9.6 \pm 0.4$ & 4 \\
\hline $081126-1$ & 5877.227 & $\ldots$ & He I & $\ldots$ & $\ldots$ & $\ldots$ \\
\hline $081126-2$ & 5877.227 & 5876.947 & He I & 3.6 & $-0.2 \pm 0.1$ & -14 \\
\hline $081126-3$ & 5877.227 & 5877.406 & He I & 4.0 & $-0.6 \pm 0.1$ & 9 \\
\hline 081127 & 5877.227 & 5877.451 & $\mathrm{He} \mathrm{I}$ & 3.8 & $-0.3 \pm 0.1$ & 11 \\
\hline 090106 & 5877.227 & $\ldots$ & $\mathrm{He}_{\mathrm{I}}$ & $\ldots$ & $\ldots$ & $\ldots$ \\
\hline 090110 & 5877.227 & $\ldots$ & He I & $\ldots$ & $\ldots$ & $\ldots$ \\
\hline 090111 & 5877.227 & $\ldots$ & He I & $\ldots$ & $\ldots$ & $\ldots$ \\
\hline 090305 & 5877.227 & 5877.130 & $\mathrm{He}_{\mathrm{I}}$ & 4.4 & $-0.5 \pm 0.1$ & -5 \\
\hline 090531 & 5877.227 & 5877.381 & He I & 3.6 & $-0.2 \pm 0.1$ & 8 \\
\hline $081126-1$ & 5891.583 & 5892.387 & $\mathrm{Na}$ I $D^{\mathrm{e}}$ & 5.1 & $-1.6 \pm 0.1$ & 41 \\
\hline$\ldots$ & 5891.583 & 5890.280 & $\mathrm{Na}$ I $D^{\mathrm{e}}$ & $\ldots$ & $\ldots$ & -66 \\
\hline
\end{tabular}


Table 5

(Continued)

\begin{tabular}{|c|c|c|c|c|c|c|}
\hline UT Date & $\lambda_{\text {lab }}(\AA)$ & $\lambda_{\text {obs }}(\AA)$ & Ion & Flux $^{\mathrm{a}}$ & $\mathrm{EW} \pm 1 \sigma(\AA)^{\mathrm{b}}$ & $v\left(\mathrm{~km} \mathrm{~s}^{-1}\right)^{\mathrm{c}}$ \\
\hline \multirow{2}{*}{$\begin{array}{l}081126-2 \\
\ldots\end{array}$} & 5891.583 & 5892.314 & $\mathrm{Na}_{\mathrm{I}} \mathrm{D}^{\mathrm{e}}$ & 5.3 & $-1.9 \pm 0.1$ & 37 \\
\hline & 5891.583 & 5890.062 & $\mathrm{Na}$ I $D^{\mathrm{e}}$ & $\ldots$ & $\ldots$ & -77 \\
\hline \multirow[t]{2}{*}{$081126-3$} & 5891.583 & 5892.286 & $\mathrm{Na}$ I $D^{\mathrm{e}}$ & 5.3 & $-1.4 \pm 0.1$ & 36 \\
\hline & 5891.583 & 5890.193 & $\mathrm{Na}$ I $D^{\mathrm{e}}$ & $\ldots$ & $\ldots$ & -71 \\
\hline \multirow{2}{*}{$\begin{array}{l}081127 \\
\ldots\end{array}$} & 5891.583 & 5892.357 & $\mathrm{Na}_{\mathrm{I}} \mathrm{D}^{\mathrm{e}}$ & 5.5 & $-2.0 \pm 0.1$ & 39. \\
\hline & 5891.583 & 5890.000 & $\mathrm{Na}$ I $D^{\mathrm{e}}$ & $\ldots$ & $\ldots$ & -81 \\
\hline \multirow{2}{*}{$\begin{array}{l}090106 \\
\ldots\end{array}$} & 5891.583 & 5892.210 & $\mathrm{Na}$ I $D^{\mathrm{e}}$ & 9.7 & $-5.8 \pm 0.7$ & 32 \\
\hline & 5891.583 & 5890.436 & $\mathrm{Na}$ I $D^{\mathrm{e}}$ & $\ldots$ & $\ldots$ & -58 \\
\hline \multirow{2}{*}{$\begin{array}{l}090110 \\
\ldots\end{array}$} & 5891.583 & 5892.171 & $\mathrm{Na}$ I $\mathrm{D}^{\mathrm{e}}$ & 6.4 & $-3.3 \pm 0.2$ & 30 \\
\hline & 5891.583 & 5890.493 & $\mathrm{Na}$ I $D^{\mathrm{e}}$ & $\ldots$ & $\ldots$ & -56 \\
\hline \multirow{2}{*}{$\begin{array}{l}090111 \\
\ldots\end{array}$} & 5891.583 & 5892.245 & $\mathrm{Na}$ I $D^{\mathrm{e}}$ & 6.9 & $-3.9 \pm 0.4$ & 34 \\
\hline & 5891.583 & 5890.496 & $\mathrm{Na}$ I $D^{\mathrm{e}}$ & $\ldots$ & $\ldots$ & -55 \\
\hline \multirow{2}{*}{$\begin{array}{l}090305 \\
090531\end{array}$} & 5891.583 & $\ldots$ & $\mathrm{Na}$ I $D^{\mathrm{f}}$ & $\ldots$ & $\ldots$ & $\ldots$ \\
\hline & 891.583 & $\ldots$ & $\mathrm{Na}$ I $D^{f}$ & $\ldots$ & $\ldots$ & $\ldots$ \\
\hline \multirow{2}{*}{$\begin{array}{l}081126-1 \\
\ldots\end{array}$} & 5897.558 & 5898.417 & $\mathrm{Na}$ I $D^{\mathrm{e}}$ & 2.2 & -1.3 & 44 \\
\hline & 5897.558 & 5895.873 & $\mathrm{Na}$ I $D^{\mathrm{e}}$ & $\ldots$ & $\ldots$ & -86 \\
\hline \multirow{2}{*}{$\begin{array}{l}081126-2 \\
\ldots\end{array}$} & 5897.558 & 5898.333 & $\mathrm{Na}$ I $\mathrm{D}^{\mathrm{e}}$ & 2.2 & -1.5 & 39 \\
\hline & 5897.558 & 5896.308 & $\mathrm{Na}$ I $D^{\mathrm{e}}$ & $\ldots$ & $\ldots$ & -64 \\
\hline \multirow{2}{*}{$\begin{array}{l}081126-3 \\
\ldots\end{array}$} & 5897.558 & 5896.938 & $\mathrm{Na}$ I $\mathrm{D}^{\mathrm{e}}$ & 5.0 & $-1.1 \pm 0.1$ & 36 \\
\hline & 5897.558 & 5896.938 & $\mathrm{Na}$ I $\mathrm{D}^{\mathrm{e}}$ & $\ldots$ & $\ldots$ & -32 \\
\hline \multirow{2}{*}{$\begin{array}{l}081127 \\
\ldots\end{array}$} & 5897.558 & 5898.398 & $\mathrm{Na}$ I $D^{\mathrm{e}}$ & 4.8 & $-1.3 \pm 0.1$ & 43 \\
\hline & 5897.558 & 5896.095 & $\mathrm{Na} \mathrm{I}^{\mathrm{e}}$ & $\ldots$ & $\ldots$ & -74 \\
\hline \multirow{2}{*}{$\begin{array}{l}090106 \\
\ldots\end{array}$} & 5897.558 & 5898.136 & $\mathrm{Na}_{\mathrm{I}} \mathrm{D}^{\mathrm{e}}$ & 7.4 & $-4.1 \pm 0.7$ & 29 \\
\hline & 5897.558 & 5896.217 & $\mathrm{Na}$ I $D^{\mathrm{e}}$ & $\ldots$ & $\ldots$ & -98 \\
\hline \multirow{2}{*}{090110} & 5897.558 & 5898.106 & $\mathrm{Na}$ I $D^{\mathrm{e}}$ & 5.9 & $-2.8 \pm 0.2$ & 28 \\
\hline & 5897.558 & 5896.452 & $\mathrm{Na}$ I $D^{\mathrm{e}}$ & $\ldots$ & & -56 \\
\hline 090111 & 5897.558 & 5898.182 & $\mathrm{Na}$ I $D^{\mathrm{e}}$ & 6.1 & $-3.0 \pm 0.4$ & 32 \\
\hline$\ldots$ & 5897.558 & 5896.669 & $\mathrm{Na}$ I $\mathrm{D}^{\mathrm{e}}$ & $\ldots$ & $\ldots$ & -45 \\
\hline 090305 & 5897.558 & $\ldots$ & $\mathrm{Na}$ I $D^{f}$ & $\ldots$ & $\cdots$ & $\ldots$ \\
\hline 090531 & 5897.558 & $\ldots$ & $\mathrm{Na}$ I $D^{f}$ & $\ldots$ & $\ldots$ & $\ldots$ \\
\hline $081126-1$ & 6302.050 & 6302.223 & [OI] & 14.1 & $-9.2 \pm 0.1$ & 8 \\
\hline $081126-2$ & 6302.050 & 6302.182 & [OI] & 13.5 & $-8.9 \pm 0.1$ & 6 \\
\hline $081126-3$ & 6302.050 & 6302.206 & [OI] & 13.9 & $-9.2 \pm 0.1$ & 7 \\
\hline 081127 & 6302.050 & 6302.102 & [OI] & 14.5 & $-9.9 \pm 0.1$ & 2 \\
\hline 090106 & 6302.050 & 6302.068 & [OI] & 19.4 & $-14.8 \pm 0.3$ & 1 \\
\hline 090110 & 6302.050 & 6302.033 & [OI] & 18.3 & $-13.7 \pm 0.1$ & -1 \\
\hline 090111 & 6302.050 & 6302.114 & [OI $]$ & 18.0 & $-13.4 \pm 0.1$ & 3 \\
\hline 090305 & 6302.050 & 6302.182 & [OI] & 7.8 & $-3.2 \pm 0.1$ & 6 \\
\hline 090531 & 6302.050 & 6302.313 & [O I $]$ & 7.8 & $-3.1 \pm 0.1$ & 13 \\
\hline 081126-1 & 6365.540 & 6365.655 & [OI] & 6.3 & $-2.1 \pm 0.1$ & 5 \\
\hline $081126-2$ & 6365.540 & 6365.721 & {$[\mathrm{O} I]$} & 6.6 & $-1.9 \pm 0.1$ & 9 \\
\hline $081126-3$ & 6365.540 & 6365.752 & {$\left[\mathrm{O}_{\mathrm{I}}\right]$} & 6.8 & $-2.2 \pm 0.1$ & 10 \\
\hline 081127 & 6365.540 & 6365.530 & {$\left[\mathrm{O}_{\mathrm{I}}\right]$} & 6.9 & $-2.3 \pm 0.1$ & -0 \\
\hline 090106 & 6365.540 & 6365.558 & [OI] & 7.6 & $-3.4 \pm 0.1$ & 1 \\
\hline 090110 & 6365.540 & 6365.507 & [OI] & 8.1 & $-3.4 \pm 0.1$ & -2 \\
\hline 090111 & 6365.540 & 6365.562 & [OI] & 7.4 & $-3.2 \pm 0.1$ & 1 \\
\hline 090305 & 6365.540 & 6365.717 & {$\left[\mathrm{O}_{\mathrm{I}}\right]$} & 5.5 & $-0.8 \pm 0.1$ & 8 \\
\hline 090531 & 6365.540 & 6365.914 & [O I] & 5.5 & $-0.9 \pm 0.1$ & 18 \\
\hline $081126-1$ & 6549.850 & 6550.278 & {$[\mathrm{~N}$ II $]$} & 3.0 & $-0.2 \pm 0.1$ & 20 \\
\hline $081126-2$ & 6549.850 & 6549.824 & {$\left[\mathrm{~N}_{\mathrm{II}}\right]$} & 3.1 & $-0.2 \pm 0.1$ & -1 \\
\hline $081126-3$ & 6549.850 & $\ldots$ & {$[\mathrm{N}$ II] } & $\ldots$ & $\ldots$ & $\ldots$ \\
\hline 081127 & 6549.850 & 6550.045 & {$[\mathrm{~N} \mathrm{II}]$} & 3.0 & $-0.1 \pm 0.1$ & 9 \\
\hline 090106 & 6549.850 & 6550.119 & {$[\mathrm{~N}$ II $]$} & 3.2 & $-0.4 \pm 0.1$ & 12 \\
\hline 090110 & 6549.850 & 6549.875 & {$[\mathrm{~N}$ II $]$} & 3.8 & $-0.4 \pm 0.1$ & 1 \\
\hline 090111 & 6549.850 & 6549.924 & {$[\mathrm{~N}$ II] } & 3.7 & $-0.3 \pm 0.1$ & 3 \\
\hline 090305 & 6549.850 & 6549.632 & {$[\mathrm{~N}$ II $]$} & 3.2 & $-0.3 \pm 0.1$ & -10 \\
\hline 090531 & 6549.850 & 6549.865 & {$[\mathrm{~N} \mathrm{II}]$} & 3.1 & $-0.2 \pm 0.1$ & 1 \\
\hline $081126-1$ & 6585.280 & 6585.279 & {$\left[\mathrm{~N}_{\mathrm{II}}\right]$} & 5.0 & $-0.6 \pm 0.1$ & 0 \\
\hline $081126-2$ & 6585.280 & 6585.366 & {$[\mathrm{~N}$ II $]$} & 5.4 & $-0.6 \pm 0.1$ & 4 \\
\hline $081126-3$ & 6585.280 & 6585.170 & {$[\mathrm{~N} \mathrm{II}]$} & 4.8 & $-0.5 \pm 0.1$ & -5 \\
\hline 081127 & 6585.280 & 6585.169 & {$[\mathrm{~N}$ II] } & 4.9 & $-0.5 \pm 0.1$ & -5 \\
\hline 090106 & 6585.280 & 6585.407 & {$[\mathrm{~N}$ II] } & 5.9 & $-1.0 \pm 0.1$ & 6 \\
\hline 090110 & 6585.280 & 6585.169 & {$\left[\mathrm{~N}_{\mathrm{II}}\right]$} & 5.1 & $-0.8 \pm 0.1$ & -5 \\
\hline 090111 & 6585.280 & 6585.377 & {$\left[\mathrm{~N}_{\mathrm{II}}\right]$} & 5.8 & $-1.0 \pm 0.1$ & 4 \\
\hline
\end{tabular}


Table 5

(Continued)

\begin{tabular}{|c|c|c|c|c|c|c|}
\hline UT Date & $\lambda_{\text {lab }}(\AA)$ & $\lambda_{\text {obs }}(\AA)$ & Ion & Flux $^{\mathrm{a}}$ & $\mathrm{EW} \pm 1 \sigma(\AA)^{\mathrm{b}}$ & $v\left(\mathrm{~km} \mathrm{~s}^{-1}\right)^{\mathrm{c}}$ \\
\hline 090305 & 6585.280 & 6584.912 & {$[\mathrm{~N} \mathrm{II}]$} & 5.1 & $-0.3 \pm 0.1$ & -17 \\
\hline 090531 & 6585.280 & 6585.067 & {$[\mathrm{~N}$ II $]$} & 4.7 & $-0.4 \pm 0.1$ & -10 \\
\hline 081126-1 & 6718.290 & 6718.621 & {$[\mathrm{~S}$ II $]$} & 3.9 & $-0.9 \pm 0.1$ & 15 \\
\hline $081126-2$ & 6718.290 & 6718.504 & {$[\mathrm{~S}$ II $]$} & 3.5 & $-1.0 \pm 0.1$ & 10 \\
\hline $081126-3$ & 6718.290 & 6718.520 & {$[\mathrm{~S}$ II $]$} & 3.7 & $-1.2 \pm 0.1$ & 10 \\
\hline 081127 & 6718.290 & 6718.518 & {$[\mathrm{~S}$ II $]$} & 3.6 & $-1.1 \pm 0.1$ & 10 \\
\hline 090106 & 6718.290 & 6718.627 & [S II] & 5.5 & $-2.6 \pm 0.1$ & 15 \\
\hline 090110 & 6718.290 & 6718.521 & [S II] & 5.2 & $-2.7 \pm 0.1$ & 10 \\
\hline 090111 & 6718.290 & 6718.600 & {$[\mathrm{~S}$ II] } & 5.5 & $-2.5 \pm 0.1$ & 14 \\
\hline 090305 & 6718.290 & 6718.376 & {$[\mathrm{~S}$ II] } & 2.8 & $-0.3 \pm 0.1$ & 4 \\
\hline 090531 & 6718.290 & 6718.280 & {$[\mathrm{~S}$ II $]$} & 3.0 & $-0.5 \pm 0.1$ & 0 \\
\hline 081126-1 & 6732.670 & & {$[\mathrm{~S} \mathrm{II}]$} & $\ldots$ & & $\ldots$ \\
\hline $081126-2$ & 6732.670 & 6733.000 & {$[\mathrm{~S}$ II $]$} & 5.0 & $-1.5 \pm 0.1$ & 15 \\
\hline $081126-3$ & 6732.670 & 6733.000 & [S II] & 5.0 & $-1.6 \pm 0.1$ & 15 \\
\hline 081127 & 6732.670 & 6732.888 & [S II] & 5.2 & $-1.7 \pm 0.1$ & 10 \\
\hline 090106 & 6732.670 & 6732.990 & {$[\mathrm{~S}$ II $]$} & 7.5 & $-4.0 \pm 0.1$ & 14 \\
\hline 090110 & 6732.670 & 6732.913 & {$[\mathrm{~S}$ II $]$} & 7.3 & $-3.4 \pm 0.1$ & 11 \\
\hline 090111 & 6732.670 & 6732.962 & {$[\mathrm{~S}$ II] } & 6.9 & $-3.5 \pm 0.1$ & 13 \\
\hline 090305 & 6732.670 & 6733.000 & {$[\mathrm{~S}$ II $]$} & 3.9 & $-0.4 \pm 0.1$ & 15 \\
\hline 090531 & 6732.670 & 6732.673 & [S II] & 4.1 & $-0.6 \pm 0.1$ & 0 \\
\hline $081126-1$ & 7157.130 & 7157.077 & {$[\mathrm{Fe} \mathrm{II}]$} & 4.7 & $-1.0 \pm 0.1$ & -2 \\
\hline $081126-2$ & 7157.130 & 7157.067 & {$[\mathrm{Fe} \mathrm{II}]$} & 4.7 & $-1.0 \pm 0.1$ & -3 \\
\hline $081126-3$ & 7157.130 & 7157.235 & {$[\mathrm{Fe} \mathrm{II}]$} & 5.5 & $-1.3 \pm 0.1$ & 4 \\
\hline 081127 & 7157.130 & 7157.214 & [Fe II] & 5.5 & $-1.3 \pm 0.1$ & 4 \\
\hline 090106 & 7157.130 & 7157.215 & {$[\mathrm{Fe} \mathrm{II}]$} & 6.0 & $-1.8 \pm 0.2$ & 4 \\
\hline 090110 & 7157.130 & 7157.215 & {$[\mathrm{Fe} \mathrm{II}]$} & 6.1 & $-2.0 \pm 0.1$ & 4 \\
\hline 090111 & 7157.130 & 7157.357 & {$[\mathrm{Fe} \mathrm{II}]$} & 5.9 & $-1.7 \pm 0.1$ & 10 \\
\hline 090305 & 7157.130 & 7157.116 & {$[\mathrm{Fe} \mathrm{II}]$} & 4.3 & $-0.6 \pm 0.1$ & -1 \\
\hline 090531 & 7157.130 & 7157.088 & {$[\mathrm{Fe} \mathrm{II}]$} & 4.4 & $-0.7 \pm 0.1$ & -2 \\
\hline 081126-1 & 7173.980 & 7174.055 & {$[\mathrm{Fe}$ II $]$} & 3.9 & $-0.3 \pm 0.1$ & 3 \\
\hline $081126-2$ & 7173.980 & 7173.893 & {$[\mathrm{Fe} \mathrm{II}]$} & 4.1 & $-0.4 \pm 0.1$ & -4 \\
\hline $081126-3$ & 7173.980 & 7173.871 & {$[\mathrm{Fe} \mathrm{II}]$} & 4.2 & $-0.5 \pm 0.1$ & -5 \\
\hline 081127 & 7173.980 & 7174.041 & {$[\mathrm{Fe} \mathrm{II}]$} & 4.2 & $-0.5 \pm 0.1$ & 3 \\
\hline 090106 & 7173.980 & 7174.076 & {$[\mathrm{Fe} \mathrm{II}]$} & 4.4 & $-0.7 \pm 0.1$ & 4 \\
\hline 090110 & 7173.980 & 7173.923 & {$[\mathrm{Fe} \mathrm{II}]$} & 4.4 & $-0.7 \pm 0.1$ & -2 \\
\hline 090111 & 7173.980 & 7173.910 & {$[\mathrm{Fe} \mathrm{II}]$} & 4.3 & $-0.6 \pm 0.1$ & -3 \\
\hline 090305 & 7173.980 & 7173.874 & {$[\mathrm{Fe} \mathrm{II}]$} & 4.1 & $-0.4 \pm 0.1$ & -4 \\
\hline 090531 & 7173.980 & 7173.596 & {$[\mathrm{Fe} \mathrm{II}]$} & 3.5 & $-0.3 \pm 0.1$ & -16 \\
\hline
\end{tabular}

Notes.

${ }^{a}$ The integrated line fluxes are given in units of $10^{-16} \mathrm{erg} \mathrm{cm}^{-2} \mathrm{~s}^{-1}$ and should only be used to calculate relative line fluxes between features in the same spectrum as our data are not photometrically calibrated and hence do not account for slit losses or non-photometric conditions.

${ }^{\mathrm{b}}$ Some lines were measured by hand and have typical uncertainties of $0.5-3 \AA$.

${ }^{c}$ These velocities are not corrected to the stellar rest frame $\left(12.3 \pm 1.5 \mathrm{~km} \mathrm{~s}^{-1}\right)$.

${ }^{\mathrm{d}}$ This line is blended or contaminated.

e This line shows both blue and redshifted components. The position of the weaker line component is reported but not the flux or EW.

${ }^{\mathrm{f}}$ This line has a P Cygni profile.

a spectral type of $\sim \mathrm{M} 4.5$, although this probably includes accretion continuum excess emission (see Section 4.2) and (2) using the latest NIR spectral type, M6, which likely has the least accretion continuum excess (see Section 4.2), although the spectral type may be slightly too late due to our use of field templates for the NIR data.

For the first estimate, we assume that the 2MASS data point $\left(K_{s}=8.77 \pm 0.02 \mathrm{mag}\right)$ has $A_{V}$ and $A_{K}=0$. From the $T_{\text {eff }}$ scale $^{19}$ for cTTS of Luhman et al. (2003), a spectral type of M4.5 gives an effective temperature of $3200 \pm 100 \mathrm{~K}$. The bolometric correction in $K$ band $\left(\mathrm{BC}_{K}\right)$ from Golimowski et al. (2004)

\footnotetext{
19 Luhman \& Muench (2008) estimate an uncertainty of $\sim 100 \mathrm{~K}$ in this
} relation. begins at spectral type $\mathrm{M} 6$, yielding $\mathrm{BC}_{K}=3.03 \pm 0.13 \mathrm{mag}$ for $\mathrm{M} 6$ and $\mathrm{BC}_{K}=3.06 \pm 0.13 \mathrm{mag}$ for M7. Although M4.5 is earlier than the beginning of this relation, the change in $\mathrm{BC}_{K}$ over one spectral type $(\Delta=0.03 \mathrm{mag})$ is small in comparison to the root mean square (rms) in the uncertainty $(0.13 \mathrm{mag})$. We therefore adopt a value of $\mathrm{BC}_{K}=3.0 \pm 0.1 \mathrm{mag}$ on the CIT photometric system. Applying the transformation of Carpenter (2001), 2MASS $K_{s}=$ CIT $K-0.024 \mathrm{mag}$, with negligible uncertainties and a color term of zero. Hence, 2MASS $K_{s}=$ $8.77 \pm 0.02 \mathrm{mag}$ transforms into CIT $K=8.79 \pm 0.02 \mathrm{mag}$. For a distance of $42 \pm 2 \mathrm{pc}$ (the kinematic distance), the distance modulus is $3.12 \pm 0.10 \mathrm{mag}$, yielding CIT $M_{K}=5.67 \pm$ $0.10 \mathrm{mag}$. Applying the bolometric correction yields $M_{\mathrm{bol}}=8.7$ $\pm 0.1 \mathrm{mag}$. Adopting the IAU standard of $M_{\mathrm{bol}, \odot}=4.75 \mathrm{mag}$, 
Table 6

Equivalent Widths of Selected Absorption Lines for TWA 30

\begin{tabular}{|c|c|c|c|c|c|c|}
\hline UT Date & $\lambda_{\text {lab }}(\AA)$ & $\lambda_{\text {obs }}(\AA)$ & Ion & Flux $^{\mathrm{a}}$ & $\mathrm{EW} \pm 1 \sigma(\AA)$ & $v\left(\mathrm{~km} \mathrm{~s}^{-1}\right)^{\mathrm{b}}$ \\
\hline 081126-1 & 5330.014 & 5330.021 & $\mathrm{Fe}_{\mathrm{I}}$ & 1.8 & $0.6 \pm 0.1$ & 0 \\
\hline $081126-2$ & 5330.014 & $\ldots$ & $\mathrm{Fe}_{\mathrm{I}}$ & $\ldots$ & $\ldots$ & $\ldots$ \\
\hline $081126-3$ & 5330.014 & 5329.688 & $\mathrm{Fe}_{\mathrm{I}}$ & 2.0 & $0.3 \pm 0.1$ & -18 \\
\hline 081127 & 5330.014 & 5329.742 & $\mathrm{Fe}_{\mathrm{I}}$ & 2.2 & $0.5 \pm 0.1$ & -15 \\
\hline 090106 & 5330.014 & $\ldots$ & $\mathrm{Fe} I$ & $\ldots$ & $\ldots$ & $\ldots$ \\
\hline 090110 & 5330.014 & 5329.991 & $\mathrm{Fe}_{\mathrm{I}}$ & 2.0 & $0.4 \pm 0.1$ & -1 \\
\hline 090111 & 5330.014 & $\ldots$ & $\mathrm{Fe}_{\mathrm{I}}$ & $\ldots$ & $\ldots$ & $\ldots$ \\
\hline 090305 & 5330.014 & 5329.659 & $\mathrm{Fe}_{\mathrm{I}}$ & 1.8 & $0.6 \pm 0.1$ & -20 \\
\hline 090531 & 5330.014 & 5329.668 & $\mathrm{Fe}_{\mathrm{I}}$ & 1.7 & $0.7 \pm 0.1$ & -19 \\
\hline 081126-1 & 5372.983 & $\ldots$ & $\mathrm{Fe}_{\mathrm{I}}$ & $\ldots$ & $\ldots$ & $\ldots$ \\
\hline $081126-2$ & 5372.983 & 5373.160 & $\mathrm{Fe}_{\mathrm{I}}$ & 1.7 & $0.3 \pm 0.1$ & 10 \\
\hline $081126-3$ & 5372.983 & 5373.200 & $\mathrm{Fe}_{\mathrm{I}}$ & 1.8 & $0.6 \pm 0.1$ & 12 \\
\hline 081127 & 5372.983 & 5373.262 & $\mathrm{Fe}_{\mathrm{I}}$ & 1.9 & $0.5 \pm 0.1$ & 16 \\
\hline 090106 & 5372.983 & $\ldots$ & $\mathrm{Fe}_{\mathrm{I}}$ & $\ldots$ & $\ldots$ & $\ldots$ \\
\hline 090110 & 5372.983 & $\ldots$ & $\mathrm{Fe}_{\mathrm{I}}$ & $\ldots$ & $\ldots$ & $\ldots$ \\
\hline 090111 & 5372.983 & $\ldots$ & Fe I & $\ldots$ & $\ldots$ & $\ldots$ \\
\hline 090305 & 5372.983 & 5373.091 & $\mathrm{Fe}_{\mathrm{I}}$ & 1.6 & $0.4 \pm 0.1$ & 6 \\
\hline 090531 & 5372.983 & 5373.176 & $\mathrm{Fe}_{\mathrm{I}}$ & 1.9 & $0.5 \pm 0.1$ & 11 \\
\hline 081126-1 & 5398.629 & 5398.770 & $\mathrm{Fe}_{\mathrm{I}}$ & 1.6 & $0.4 \pm 0.1$ & 8 \\
\hline $081126-2$ & 5398.629 & $\ldots$ & $\mathrm{Fe}_{\mathrm{I}}$ & $\ldots$ & $\ldots$ & $\ldots$ \\
\hline $081126-3$ & 5398.629 & $\ldots$ & $\mathrm{Fe}_{\mathrm{I}}$ & $\ldots$ & $\ldots$ & $\ldots$ \\
\hline 081127 & 5398.629 & $\ldots$ & $\mathrm{Fe}_{\mathrm{I}}$ & $\ldots$ & $\ldots$ & $\ldots$ \\
\hline 090106 & 5398.629 & $\ldots$ & $\mathrm{Fe}_{\mathrm{I}}$ & $\ldots$ & $\ldots$ & $\ldots$ \\
\hline 090110 & 5398.629 & $\ldots$ & $\mathrm{Fe}_{\mathrm{I}}$ & $\ldots$ & $\ldots$ & $\ldots$ \\
\hline 090111 & 5398.629 & 5398.521 & Fe I & 1.9 & $0.5 \pm 0.1$ & -6 \\
\hline 090305 & 5398.629 & 5398.597 & $\mathrm{Fe}_{\mathrm{I}}$ & 1.6 & $0.4 \pm 0.1$ & -2 \\
\hline 090531 & 5398.629 & $\ldots$ & $\mathrm{Fe}_{\mathrm{I}}$ & $\ldots$ & $\ldots$ & $\ldots$ \\
\hline $081126-1$ & 5407.278 & $\ldots$ & $\mathrm{Fe}_{\mathrm{I}}$ & $\ldots$ & $\ldots$ & $\ldots$ \\
\hline $081126-2$ & 5407.278 & $\ldots$ & $\mathrm{Fe}_{\mathrm{I}}$ & $\ldots$ & $\ldots$ & $\ldots$ \\
\hline $081126-3$ & 5407.278 & $\ldots$ & $\mathrm{Fe}_{\mathrm{I}}$ & $\ldots$ & $\ldots$ & $\ldots$ \\
\hline 081127 & 5407.278 & 5407.324 & $\mathrm{Fe}_{\mathrm{I}}$ & 2.1 & $0.3 \pm 0.1$ & 3 \\
\hline 090106 & 5407.278 & $\ldots$ & $\mathrm{Fe}_{\mathrm{I}}$ & $\ldots$ & $\ldots$ & $\ldots$ \\
\hline 090110 & 5407.278 & 5407.136 & $\mathrm{Fe}_{\mathrm{I}}$ & 2.5 & $0.3 \pm 0.1$ & -8 \\
\hline 090111 & 5407.278 & $\ldots$ & $\mathrm{Fe}_{\mathrm{I}}$ & $\ldots$ & $\ldots$ & $\ldots$ \\
\hline 090305 & 5407.278 & 5407.266 & Fe I & 2.0 & $0.4 \pm 0.1$ & -1 \\
\hline 090531 & 5407.278 & 5407.266 & $\mathrm{Fe}_{\mathrm{I}}$ & 2.0 & $0.4 \pm 0.1$ & -1 \\
\hline $081126-1$ & 5411.276 & 5411.733 & Cr I & 2.5 & $0.3 \pm 0.1$ & 25 \\
\hline $081126-2$ & 5411.276 & 5411.443 & Cr I & 2.3 & $0.5 \pm 0.1$ & 9 \\
\hline $081126-3$ & 5411.276 & $\ldots$ & Cr I & $\ldots$ & $\ldots$ & $\ldots$ \\
\hline 081127 & 5411.276 & 5411.307 & Cr I & 2.4 & $0.3 \pm 0.1$ & 2 \\
\hline 090106 & 5411.276 & $\ldots$ & Cri & $\ldots$ & $\ldots$ & $\ldots$ \\
\hline 090110 & 5411.276 & 5411.713 & $\mathrm{Cr} \mathrm{I}$ & 2.3 & $0.5 \pm 0.1$ & 24 \\
\hline 090111 & 5411.276 & $\ldots$ & $\mathrm{CrI}$ & $\ldots$ & $\ldots$ & $\ldots$ \\
\hline 090305 & 5411.276 & $\ldots$ & Cr I & $\ldots$ & $\ldots$ & $\ldots$ \\
\hline 090531 & 5411.276 & $\ldots$ & Cr I & $\ldots$ & $\ldots$ & $\ldots$ \\
\hline 081126-1 & 6123.912 & 6124.077 & $\mathrm{CaI}$ & 2.6 & $0.5 \pm 0.1$ & 8 \\
\hline $081126-2$ & 6123.912 & 6123.961 & $\mathrm{CaI}$ & 2.8 & $0.4 \pm 0.1$ & 2 \\
\hline $081126-3$ & 6123.912 & 6124.128 & $\mathrm{CaI}$ & 2.8 & $0.3 \pm 0.1$ & 11 \\
\hline 081127 & 6123.912 & 6124.148 & $\mathrm{CaI}$ & 2.8 & $0.3 \pm 0.1$ & 12 \\
\hline 090106 & 6123.912 & $\ldots$ & $\mathrm{Ca} \mathrm{I}$ & $\ldots$ & $\ldots$ & $\ldots$ \\
\hline 090110 & 6123.912 & 6123.958 & $\mathrm{CaI}$ & 2.8 & $0.4 \pm 0.1$ & 2 \\
\hline 090111 & 6123.912 & 6124.206 & $\mathrm{CaI}$ & 2.7 & $0.4 \pm 0.1$ & 14 \\
\hline 090305 & 6123.912 & 6123.981 & $\mathrm{CaI}$ & 2.8 & $0.3 \pm 0.1$ & 3 \\
\hline 090531 & 6123.912 & 6123.982 & $\mathrm{Ca} \mathrm{I}$ & 2.7 & $0.4 \pm 0.1$ & 3 \\
\hline $081126-1$ & 6709.660 & 6710.010 & Li I & 3.8 & $0.6 \pm 0.1$ & 16 \\
\hline $081126-2$ & 6709.660 & 6709.928 & Li I & 3.7 & $0.7 \pm 0.1$ & 12 \\
\hline $081126-3$ & 6709.660 & 6709.953 & Li I & 3.8 & $0.6 \pm 0.1$ & 13 \\
\hline 081127 & 6709.660 & 6709.794 & Li I & 3.9 & $0.6 \pm 0.1$ & 6 \\
\hline 090106 & 6709.660 & 6709.946 & Li I & 3.0 & $0.5 \pm 0.1$ & 13 \\
\hline 090110 & 6709.660 & 6709.913 & Li I & 3.0 & $0.4 \pm 0.1$ & 11 \\
\hline 090111 & 6709.660 & 6709.995 & Li I & 2.9 & $0.5 \pm 0.1$ & 15 \\
\hline 090305 & 6709.660 & 6709.753 & Li I & 3.1 & $0.8 \pm 0.1$ & 4 \\
\hline 090531 & 6709.660 & 6709.769 & Li I & 3.2 & $0.8 \pm 0.1$ & 5 \\
\hline
\end{tabular}


Table 6

(Continued)

\begin{tabular}{|c|c|c|c|c|c|c|}
\hline UT Date & $\lambda_{\text {lab }}(\AA)$ & $\lambda_{\text {obs }}(\AA)$ & Ion & Flux $^{\mathrm{a}}$ & $\mathrm{EW} \pm 1 \sigma(\AA)$ & $v\left(\mathrm{~km} \mathrm{~s}^{-1}\right)^{\mathrm{b}}$ \\
\hline 081126-1 & 7701.093 & 7701.091 & $\mathrm{~K}_{\mathrm{I}}$ & 3.1 & $0.9 \pm 0.1$ & 0 \\
\hline $081126-2$ & 7701.093 & 7701.064 & $\mathrm{~K}_{\mathrm{I}}$ & 3.1 & $0.9 \pm 0.1$ & -1 \\
\hline $081126-3$ & 7701.093 & 7701.015 & $\mathrm{~K}_{\mathrm{I}}$ & 3.1 & $0.9 \pm 0.1$ & -3 \\
\hline 081127 & 7701.093 & 7700.945 & $\mathrm{~K}_{\mathrm{I}}$ & 3.3 & $0.7 \pm 0.1$ & -6 \\
\hline 090106 & 7701.093 & 7701.001 & $\mathrm{~K}_{\mathrm{I}}$ & 5.4 & $-1.4 \pm 0.1^{\mathrm{c}}$ & -4 \\
\hline 090110 & 7701.093 & 7700.869 & $\mathrm{~K}_{\mathrm{I}}$ & 5.1 & $-1.2 \pm 0.1^{\mathrm{c}}$ & -9 \\
\hline 090111 & 7701.093 & 7700.895 & $\mathrm{~K}_{\mathrm{I}}$ & 5.1 & $-1.1 \pm 0.1^{\mathrm{c}}$ & -8 \\
\hline 090305 & 7701.093 & 7701.001 & $\mathrm{~K}_{\mathrm{I}}$ & 2.7 & $1.3 \pm 0.1$ & -4 \\
\hline 090531 & 7701.093 & 7700.891 & $\mathrm{~K}_{\mathrm{I}}$ & 2.5 & $1.4 \pm 0.1$ & -8 \\
\hline $081126-1$ & 8185.505 & 8185.689 & $\mathrm{Na} I$ & 3.2 & $1.1 \pm 0.1$ & 7 \\
\hline $081126-2$ & 8185.505 & 8185.622 & Na I & 3.3 & $0.9 \pm 0.1$ & 4 \\
\hline $081126-3$ & 8185.505 & 8185.611 & $\mathrm{NaI}$ & 3.2 & $1.0 \pm 0.1$ & 4 \\
\hline 081127 & 8185.505 & 8185.532 & $\mathrm{NaI}$ & 3.8 & $1.0 \pm 0.1$ & 1 \\
\hline 090106 & 8185.505 & 8185.748 & $\mathrm{Na}$ & 3.2 & $1.0 \pm 0.1$ & 9 \\
\hline 090110 & 8185.505 & 8185.533 & $\mathrm{Na}$ & 3.8 & $1.0 \pm 0.1$ & 1 \\
\hline 090111 & 8185.505 & 8185.732 & $\mathrm{NaI}$ & 3.3 & $1.0 \pm 0.1$ & 8 \\
\hline 090305 & 8185.505 & 8185.439 & $\mathrm{Na}$ & 3.9 & $0.9 \pm 0.1$ & -2 \\
\hline 090531 & 8185.505 & 8185.633 & $\mathrm{Na}$ & 3.3 & $0.9 \pm 0.1$ & 5 \\
\hline
\end{tabular}

Notes.

${ }^{\text {a }}$ The integrated line fluxes are given in units of $10^{-16} \mathrm{erg} \mathrm{cm}^{-2} \mathrm{~s}^{-1}$ and should only be used to calculate relative line fluxes between features in the same spectrum as our data are not photometrically calibrated and hence do not account for slit losses or non-photometric conditions.

$\mathrm{b}$ These velocities are not corrected to the stellar rest frame $\left(12.3 \pm 1.5 \mathrm{~km} \mathrm{~s}^{-1}\right)$.

${ }^{\mathrm{c}}$ This line is seen in emission.

Table 7

Derived NIR Magnitudes ${ }^{\mathrm{a}}$, Colors $^{\mathrm{a}}$, Spectral Types, and $A_{v}$ Values of TWA 30

\begin{tabular}{lccccccccc}
\hline \hline UT Date $^{\mathrm{b}}$ & $J$ & $H$ & $K_{S}$ & $J-H$ & $H-K_{s}$ & $J-K_{s}$ & NIR SpT $^{\mathrm{c}}$ & $A_{v}$ & Notes \\
\hline 990324 & 9.64 & 9.03 & 8.77 & 0.61 & 0.27 & 0.88 & $\ldots$ & $\ldots$ & 2MASS PSC \\
081204 & 10.2 & 9.3 & 8.8 & 0.91 & 0.43 & 1.34 & M5 & 2.5 & SpeX SXD \\
081215 & 10.5 & 9.6 & 9.2 & 0.85 & 0.41 & 1.25 & M5 & 2.2 & SpeX SXD \\
090202 & 9.8 & 9.0 & 8.7 & 0.75 & 0.35 & 1.09 & M4.5 & 1.5 & SpeX SXD \\
090514 & 11.3 & 10.2 & 9.6 & 1.09 & 0.61 & 1.71 & M5.5 & 4.2 & SpeX SXD \\
090515 & 11.7 & 10.5 & 9.7 & 1.23 & 0.75 & 1.98 & M6 & 5.6 & SpeX SXD \\
090520 & 12.7 & 11.0 & 10.0 & 1.68 & 0.95 & 2.63 & M6 & 9.0 & SpeX SXD \\
090616 & 10.6 & 9.6 & 9.2 & 0.91 & 0.47 & 1.39 & M5 & 2.6 & SpeX SXD \\
090628 & 10.6 & 9.7 & 9.2 & 0.96 & 0.49 & 1.45 & $\ldots$ & $\ldots$ & SpeX prism \\
090629 & 10.5 & 9.6 & 9.1 & 0.99 & 0.48 & 1.47 & $\ldots$ & $\ldots$ & SpeX prism \\
090629 & 10.5 & 9.5 & 9.1 & 0.93 & 0.48 & 1.41 & M5 & 2.7 & SpeX SXD \\
\hline
\end{tabular}

Notes.

a All magnitudes and colors are derived using 2MASS filters for spectrophotometry on the NIR spectra taken in SXD or prism mode (as indicated) with the exception of the epoch marked "2MASS PSC," which is photometry. In photometric conditions, the derived absolute spectrophotometry, flux calibrated with nearby A0 V stars, are accurate to $\sim 10 \%$, while the derived relative spectrophotometry (colors) are accurate to a few percent (Rayner et al. 2009). For a full description, see Section 3.3.1.

${ }^{\mathrm{b}} \mathrm{UT}$ dates are recorded as YYMMDD.

${ }^{c}$ All NIR SpT and $A_{v}$ measurements were determined by dereddening the spectra to field M4-M8 templates and determining the best match, see Section 3.3.1.

$\log L_{\mathrm{bol}} / L_{\mathrm{bol}, \odot}=-1.58 \pm 0.04 \mathrm{dex}$. With this $T_{\text {eff }}$ and $L_{\mathrm{bol}}$, the evolutionary models of Baraffe et al. (1998) give an age of $10 \mathrm{Myr}$ and a mass of $0.16 M_{\odot}$.

For the second estimate, we use the latest NIR spectral type derived, M6, in 2009 May 14 and 20 with the corresponding 2MASS $K_{s}$ and $A_{V}$ estimates listed in Table 7. Using $A_{K} /$ $A_{V}=0.112$ (Rieke \& Lebofsky 1985) and the same CIT $K$ transformation as listed above to correct the NIR magnitudes of these two epochs, we find CIT $K\left(A_{K}=0\right)=9.05 \pm 0.04 \mathrm{mag}$. With the same distance modulus as above, CIT $M_{K}=5.93 \pm$ $0.11 \mathrm{mag}$. The $\mathrm{BC}_{K}$ correction from Golimowski et al. (2004) for an M6 is $3.03 \pm 0.13 \mathrm{mag}$, yielding $M_{\mathrm{bol}}=9.0 \pm 0.2 \mathrm{mag}$ and $\log L_{\mathrm{bol}} / L_{\mathrm{bol}, \odot}=-1.70 \pm 0.08$ dex. The $T_{\text {eff }}$ scale of Luhman et al. (2003) for an M6 gives $T_{\text {eff }}=3000 \pm 100 \mathrm{~K}$. With this
$T_{\text {eff }}$ and $L_{\text {bol }}$, the evolutionary models of Baraffe et al. (1998) give an age of $4 \mathrm{Myr}$ and a mass of $0.08 M_{\odot}$.

As these two spectral types and NIR photometric measurements cover nearly the full range of our measurements, the age of TWA 30 likely lies somewhere between these extremes. The mass inferred from the optical data is likely an overestimation, due to the inclusion of accretion luminosity, while the age inferred from the NIR data is likely an underestimation due to our use of field templates instead of young templates to infer the NIR spectral type. Given these uncertainties, we adopt an age of $7 \pm 3 \mathrm{Myr}$ and a mass of $0.12 \pm 0.04 M_{\odot}$. This age estimate agrees with the estimated age of the TWA ( $\sim 8 \mathrm{Myr})$, and the mass estimate places it near the substellar boundary at $\sim 0.08 M_{\odot}($ Chabrier \& Baraffe 2000). 

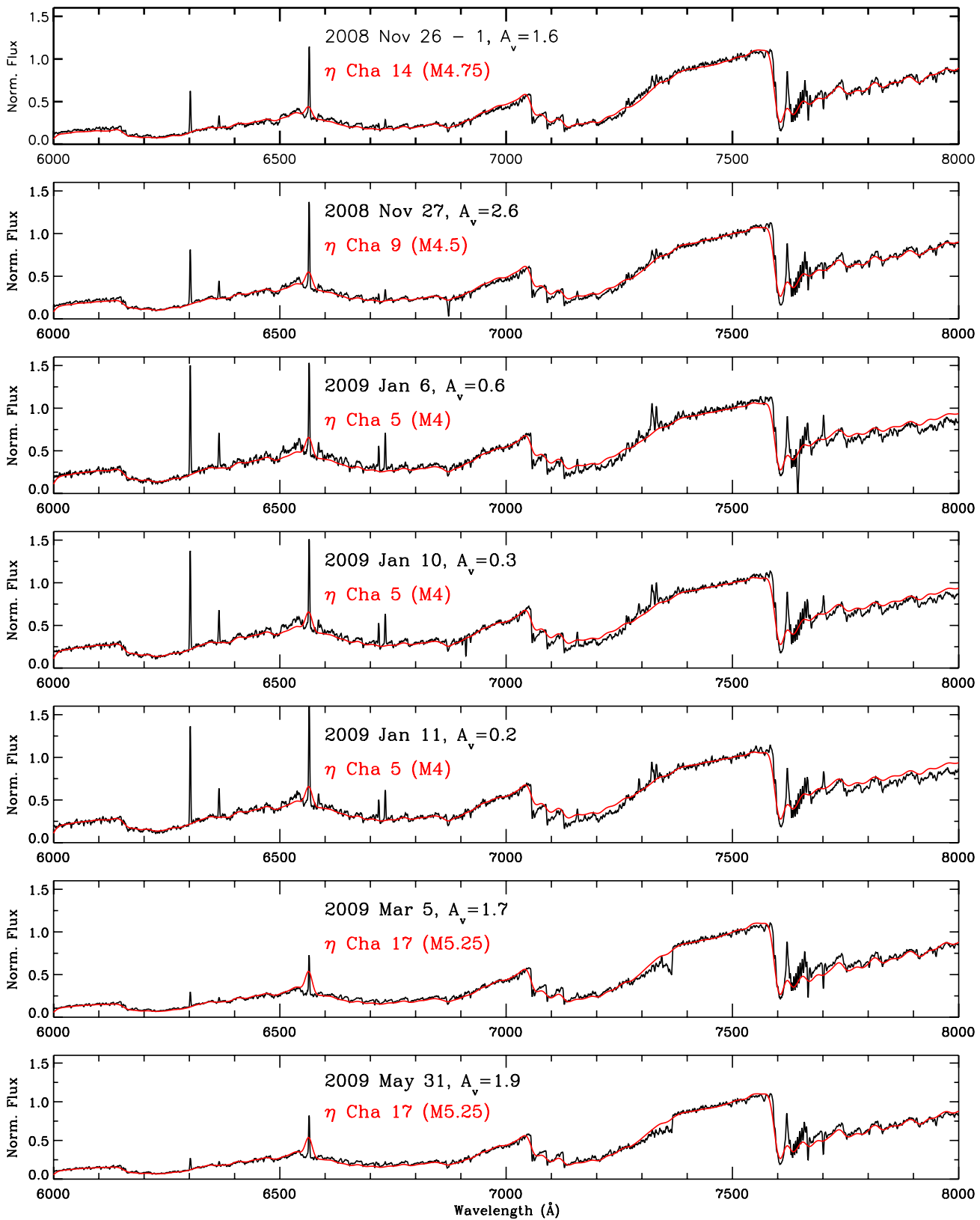

Figure 6. Overlays of the MagE optical spectra of TWA 30 (black; $R \approx 4100$ ) at each stated epoch with optical $\eta$ Cha templates (red; $R \approx 1000 ;$ Luhman \& Steeghs 2004), which provided the closest match for each epoch (see Section 3.2.1). The spectra have been dereddened by the stated amount to provide the best fit. All spectra have been normalized at $7500 \AA$.

(A color version of this figure is available in the online journal.)

\section{5. $X$-ray Activity}

The RASS Faint Source Catalog lists an X-ray detection of $(2.5 \pm 1.0) \times 10^{-2}$ counts $\mathrm{s}^{-1}$ at a distance of $17^{\prime \prime} .6$ from the 2MASS epoch position of TWA 30. The hardness ratio, HR1, is $(A-B) /(A+B)$ where band- $A$ covers $0.1-0.4 \mathrm{keV}$ (soft X-rays) and band- $B$ covers $0.5-2.0 \mathrm{keV}$ (hard $\mathrm{X}$-rays). The conversion factor, CF, from counts $\mathrm{s}^{-1}$ to X-ray flux $\left(\mathrm{erg} \mathrm{cm}^{-2} \mathrm{~s}^{-1}\right.$ ) for RASS data of late-type stars is derived by Fleming et al. (1995) as $\mathrm{CF}=(8.31+5.30 \times \mathrm{HR} 1) \times 10^{-12} \mathrm{erg} \mathrm{cm}^{-2} \mathrm{~s}^{-1}$. The $\mathrm{CF}$ for TWA 30 is thus $7.25 \times 10^{-12} \mathrm{erg} \mathrm{cm}^{-2} \mathrm{~s}^{-1}$ and when combined with the RASS count rate, yields an X-ray flux of $F_{X}=$ $1.8 \times 10^{-13} \mathrm{erg} \mathrm{cm}^{-2} \mathrm{~s}^{-1}$. With an adopted distance of $42 \mathrm{pc}$, we then derive $\log L_{X}=28.6$ dex. From the average bolometric luminosity calculated above $\left(\log L_{\mathrm{bol}} / L_{\mathrm{bol}, \odot}=\right.$
$-1.64 \pm 0.09 \mathrm{dex} ; L_{\mathrm{bol}}=8.8 \times 10^{31} \mathrm{erg} \mathrm{s}^{-1}$, assuming $L_{\mathrm{bol}, \odot}=$ $3.85 \times 10^{33} \mathrm{erg} \mathrm{s}^{-1}$ ), this yields $\log L_{X} / L_{\text {bol }}=-3.34$ dex. For early to mid-M dwarfs, $\log L_{X} / L_{\mathrm{bol}}$ saturates at $\sim-3$ dex (Delfosse et al. 1998). For T Tauri stars, both accreting and nonaccreting, down into the $\mathrm{BD}$ domain, $\log L_{X} / L_{\mathrm{bol}}$ ranges from $\sim-3$ to -4 dex (e.g., the Taurus XMM survey; Güdel et al. 2007), which the value of TWA 30 falls into, i.e., is typical.

\section{DISCUSSION}

\subsection{The Disk Fraction of the TWA}

Our observations provide clear evidence for the presence of an actively accreting disk and outflow around TWA 30; however, it is not the only disk-bearing low-mass source in the TWA. Low et al. (2005) measured a $25 \%$ disk fraction from a survey 

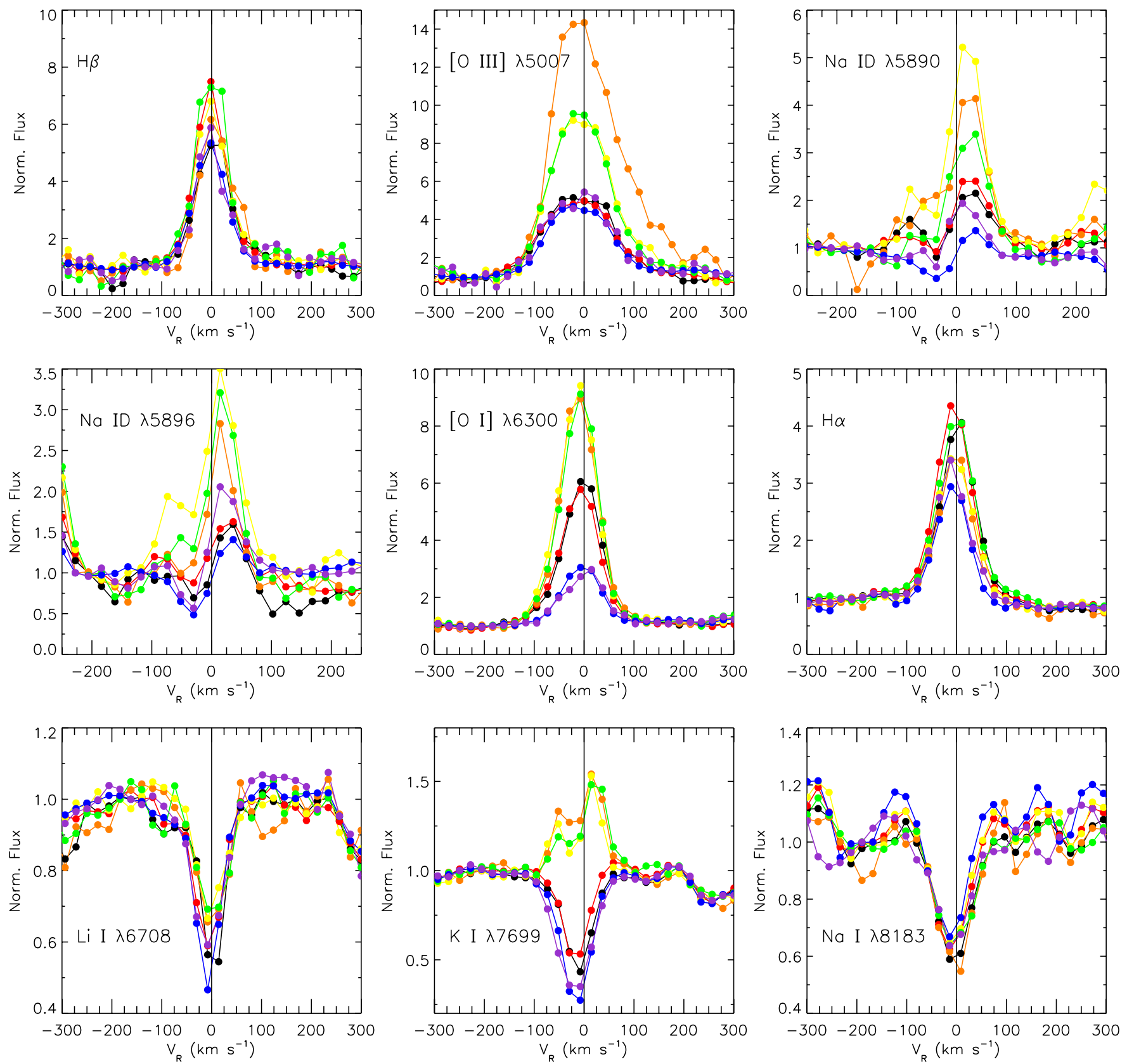

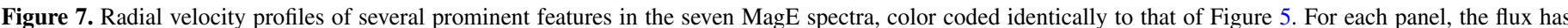

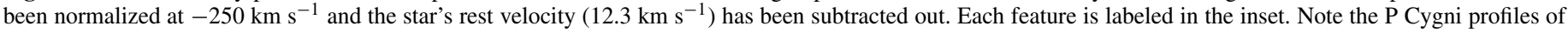
the Na I D lines in the 2009 March and May data and that the K I line goes into emission in the three 2009 January epochs.

(A color version of this figure is available in the online journal.)

of 24 candidate TWA members observed from Spitzer Space Telescope mid-IR measurements, including two M1 dwarfs (TWA 7 and TWA 13, the latter of which has been discounted by Plavchan et al. 2009). Other M dwarf members-TWA 14 (M0.5) and TWA 5A (M2.5) - have indications of passive circumstellar disks such as broad asymmetric Balmer emission (Muzerolle et al. 2001; Mohanty et al. 2003) but lack NIR excess emission (however, Jayawardhana et al. 1999 find a modest $10 \mu \mathrm{m}$ excess emission for TWA 5A). Two of three substellar systems observed in the mid-IR-TWA 28 and TWA 27A (2M1207A), and possibly TWA 27B (Mohanty et al. 2003; Sterzik et al. 2004; Riaz et al. 2006; Morrow et al. 2008; Riaz \& Gizis 2008; Herczeg et al. 2009)—also exhibit signatures of IR excess, accretion, and outflow, indicating that the same processes occur in low-mass stars and brown dwarfs (Whelan et al. 2007; Herczeg et al. 2009).

With the addition of TWA 30 as a disk-bearing low-mass member of the TWA, we updated the disk fraction of the TWA considering the following systems listed in Mamajek (2005) as bona fide members: TWA 1-11, 13-16, 20, 21, 23, and 25-28 (see Torres et al. 2008 and Lawson \& Crause 2005 for slightly different membership lists). While we consider TWA 29 (DENIS J124514.1-442907) to be a potential member, no midIR observations have yet been reported so we do not include it in our census. Of these systems, many of which are known binaries (e.g., Brandeker et al. 2003), mid-IR observations (listed above) have confirmed circumstellar disks around TWA 1, 3AB, 4AB, 7, 11A, 27A(B), and 28 (shown in R.A./decl. 


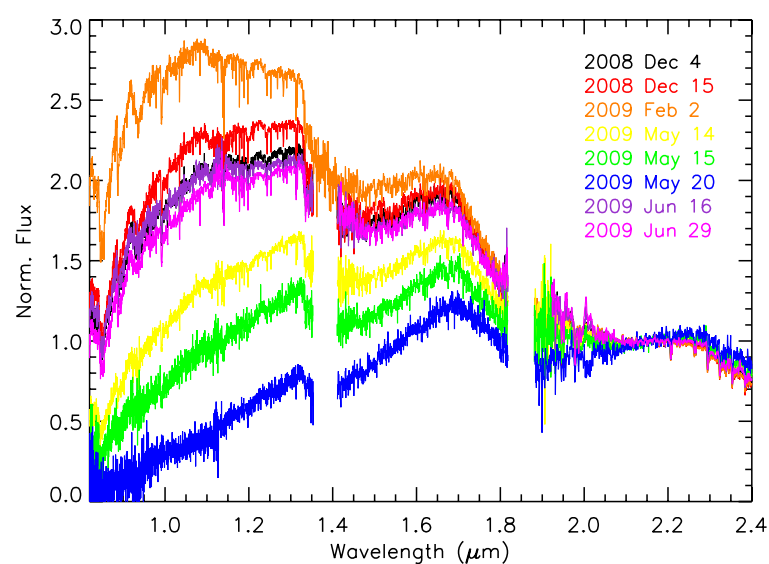

Figure 8. SpeX SXD spectra of TWA 30 at the stated epochs. All spectra have been normalized at $2.15 \mu \mathrm{m}$. The spectra have not been dereddened. The spectrophotometry and colors calculated from these data are listed in Table 7.

(A color version of this figure is available in the online journal.)

space in Figure 12). Including TWA 30 increases the diskbearing population to 8 of 23 systems, or $35_{-8}^{+11} \%$. This is a $40 \%$ increase (at $\gtrsim 1 \sigma$ ) to the estimate provided by Low et al. (2005).

\subsection{An Inclined Stellar Axis to an Edge-on Disk?}

There are seven striking features of the TWA 30 system that any scenario must explain:

1. The presence of a large number of FELs indicative of jets, with line-center velocities both blue and redshifted with respect to the stellar RV but by very small amounts (generally $\lesssim 10 \mathrm{~km} \mathrm{~s}^{-1}$ );

2. Temporal variations in the NIR colors and spectrum, with increasing reddening associated with decreasing NIR flux;

3. Temporal variations in the reddening of the optical spectrum as well;

4. Temporal variations in the underlying optical and NIR spectral types inferred after reddening effects are removed, with the earliest spectral type (optical: M4, NIR: M4.5-noncontemporaneous) associated with the lowest reddening and with the latest type (optical: M5.25, NIR: M6-noncontemporaneous) associated with the highest reddening;

5. Changes in the EWs of the optical photospheric absorption lines of $\mathrm{Li} \mathrm{I}$ and $\mathrm{KI}$, with the least absorption when the optical spectral type is earliest;

6. The sporadic appearance of $\mathrm{P}$ Cygni profiles in the $\mathrm{Na}$ $\mathrm{D}$ resonance doublet, and more weakly, in $\mathrm{H} \alpha$, with the $\mathrm{P}$ Cygni profiles being weakest/absent when the absorption in the Li I and $\mathrm{K}_{\mathrm{I}}$ lines mentioned above is least; and

7. Variations in the EWs of the FELs, with the smallest widths appearing in the last epochs of optical data when the P Cygni profiles are strongest.

\subsubsection{Evidence from FELs for an Edge-on Disk}

In regards to the first point, jets from accreting cTTS reach velocities $^{20}$ of order $200-300 \mathrm{~km} \mathrm{~s}^{-1}$. If the FELs in TWA 30 are assumed to trace such a jet, as in other cTTS, then their very low apparent velocities imply a jet that is nearly aligned with the plane of the sky. This is supported by the very low velocities

\footnotetext{
20 The jet velocity is expected to scale roughly as the escape velocity from the object (Masciadri \& Raga 2004), which is very similar for a $0.5 M_{\odot}$ fiducial cTTS and the $\sim 0.12 M_{\odot}$ TWA 30 .
}

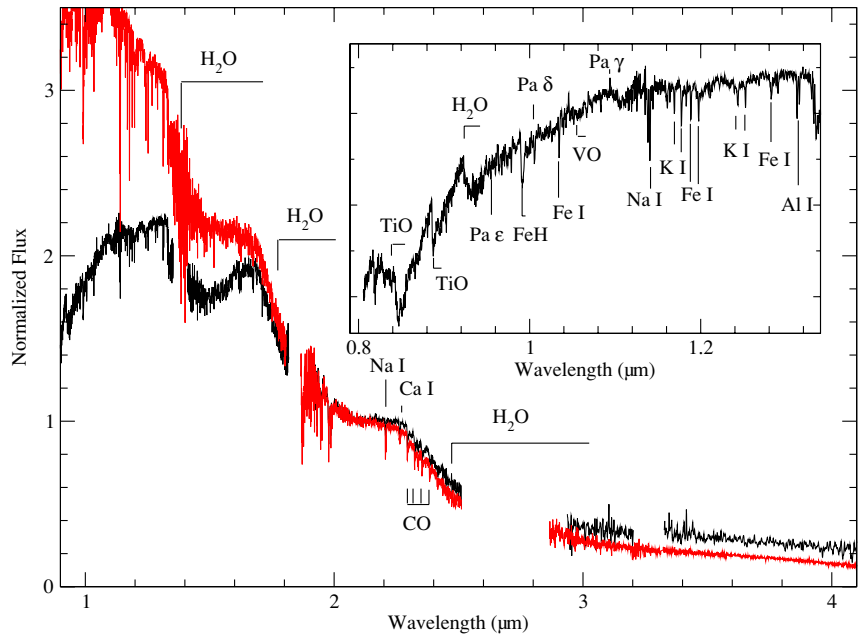

Figure 9. Combined SXD and LXD SpeX spectra of TWA 30 (black) obtained on 2008 December 4-5 UT shown in comparison to the M5 V field template Gl 51 (red). The spectrum of TWA 30 has not been dereddened here or in the inset. Both spectra have been normalized at $2.1 \mu \mathrm{m}$.

(A color version of this figure is available in the online journal.)

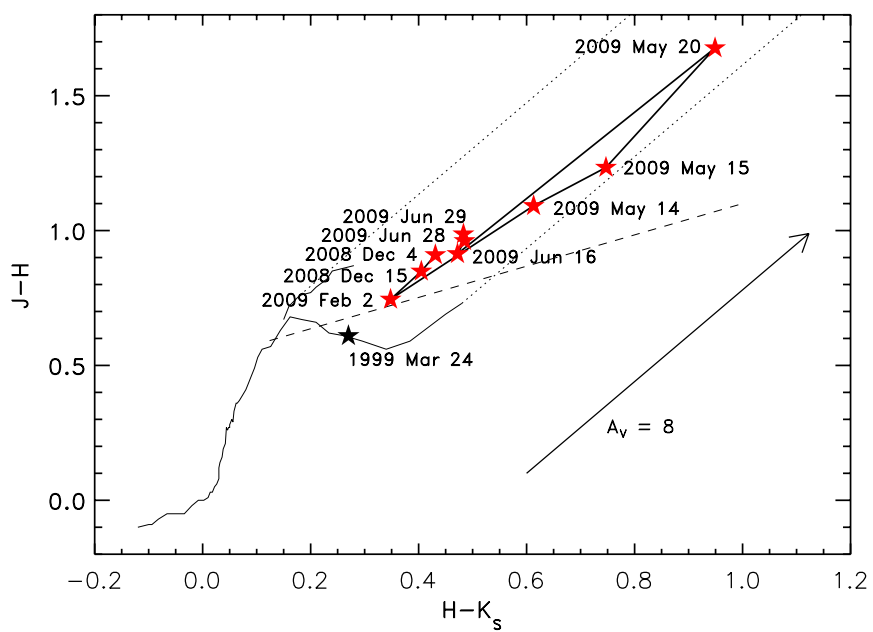

Figure 10. NIR colors of TWA 30 at each epoch of our SpeX data (red stars see Table 7) and one epoch (black star; 1999 March 24 UT) from the 2MASS PSC. The dwarf track ( $\geqslant$ M0 from Leggett 1992; earlier types from Kenyon \& Hartmann 1995) is the lower solid line and the giant track (Bessell \& Brett 1988) is the upper solid line, the vectors for reddened dwarfs are shown as two dotted lines, the cTTS locus (Meyer et al. 1997) is shown as a single dashed line, and the arrow at the lower right indicates a reddening of $A_{v}=8$. Both the dwarf and giant tracks have been transformed to the CIT photometric system, which is similar to the 2MASS system (Carpenter 2001). The red stars are connected by a solid straight line to delineate the sequence of observations.

(A color version of this figure is available in the online journal.)

seen even in the [N II] $6583 \AA$ line, which invariably exhibits only a high velocity component in cTTS (Hirth et al. 1997). By extension, the accretion disk, perpendicular to the jet, must be seen very close to edge-on. This conclusion is further supported by the presence of both blue and redshifted FELs; significant deviations from an edge-on viewing angle in cTTS produce only blueshifted FELs, as the disk obscures the receding redshifted jet lobe (e.g., Whelan et al. 2009). Further evidence for an edge-on geometry from other signatures is discussed further below.

\subsubsection{Evidence for Stellar Reddening due to Disk Dust}

The occasional yet significant reddening of the NIR spectrum (point 2), accompanied by a simultaneous dimming of the star in NIR bands, is most easily explained as stellar reddening and 
Table 8

Selected Values of MagE Optical Data for TWA 30

\begin{tabular}{|c|c|c|c|c|c|c|c|c|}
\hline UT Date $^{\mathrm{a}}$ & Opt SpT ${ }^{b}$ & $A_{V}$ & $\mathrm{H} \alpha^{\mathrm{c}}$ & {$\left[\mathrm{O}\right.$ I] $\lambda 6300^{\mathrm{c}}$} & {$\left[\mathrm{N}\right.$ II] $\lambda 6583^{\mathrm{c}}$} & {$[\mathrm{S} \mathrm{II}] \lambda 6716^{\mathrm{c}}$} & $\operatorname{Li}$ I $\lambda 6708^{c}$ & KI $\lambda 7699^{c}$ \\
\hline 081126-1 & M4.75 & 1.6 & -8.0 & -9.2 & -0.6 & -0.9 & 0.6 & 0.9 \\
\hline 081127 & M4.5 & 2.6 & -8.1 & -9.9 & -0.5 & -1.1 & 0.6 & 0.7 \\
\hline 090106 & M4 & 0.6 & -6.8 & $-14.8 \pm 0.3$ & -1.0 & -2.6 & 0.5 & -1.4 \\
\hline 090110 & M4 & 0.3 & -6.8 & -13.7 & -0.8 & -2.7 & 0.4 & -1.2 \\
\hline 090111 & M4 & 0.2 & -8.0 & -13.4 & -1.0 & -2.5 & 0.5 & -1.1 \\
\hline 090305 & M5.25 & 1.7 & -4.6 & -3.2 & -0.3 & -0.3 & 0.8 & 1.3 \\
\hline 090531 & M5.25 & 1.9 & -5.6 & -3.1 & -0.4 & -0.5 & 0.8 & 1.4 \\
\hline
\end{tabular}

Notes.

${ }^{\text {a }}$ UT dates are recorded as YYMMDD.

${ }^{\mathrm{b}}$ These spectral types were determined from the dereddened data, described in Section 3.2.1 and shown graphically in Figure 6.

${ }^{\mathrm{c}}$ The EW of the stated feature given in $\AA$. The $1 \sigma$ uncertainty is $0.1 \AA$ unless otherwise noted.

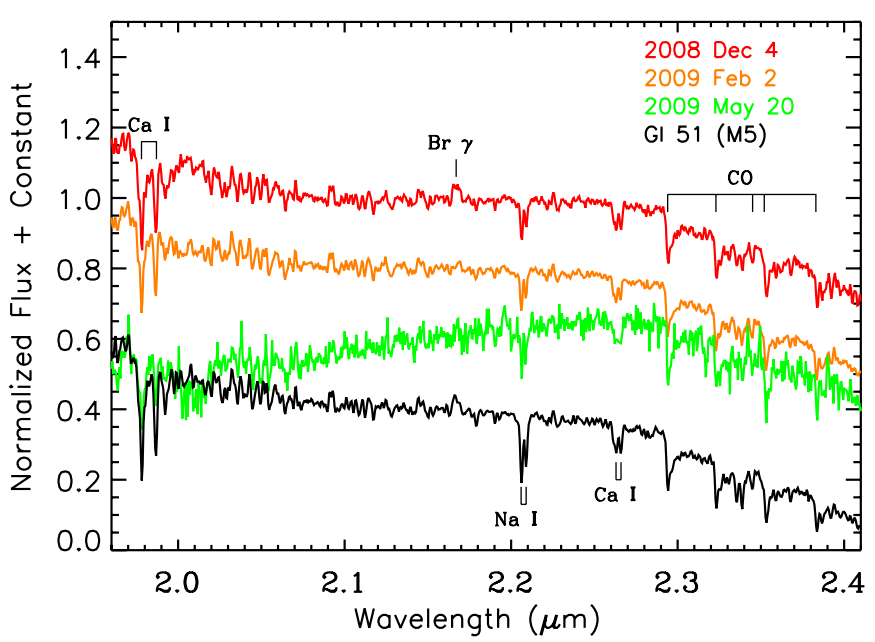

Figure 11. SpeX SXD spectra in the $K$-band region of TWA 30 at the three stated epochs (red, orange, green) shown in comparison to the M5 V field template Gl 51 (black). All spectra have been normalized at $2.15 \mu \mathrm{m}$ and are separated by constants of 0.2 along the $y$-axis. Note that the alkali doublet $\mathrm{NaI}$ is much stronger in the M5 template than in the spectra of TWA 30. Br $\gamma$ is weakly present in the 2008 December 4 UT spectrum and in the Gl 51 template but is absent in the 2009 February 2 and 2009 May 20 data.

(A color version of this figure is available in the online journal.)

occultation by disk dust within the line of sight. In the edge-on case, this dust may be entrained with the gas at the base of the outflow/accretion funnel flow near the inner edge of the disk, or signify a disk warp produced in this region by the interaction of a tilted stellar magnetosphere with the disk inner edge, as proposed for the cTTS AA Tau (Bouvier et al. 1999, 2003, 2007).

Similarly, the variable reddening observed in the optical spectrum (point 3) may then also be interpreted as extinction/ reddening caused by disk dust in the line of sight. Unfortunately, we do not have simultaneous optical photometry to verify this, as possible in the NIR. Nevertheless, the similar relationship between reddening and spectral type in the optical and NIR supports this conclusion, as described further below.

\subsubsection{Evidence for Accretion-related Veiling and Variability}

In both the optical and NIR, the underlying spectral type varies by similar amounts after the reddening effects are accounted for (point 4): from M4 to M5.25 in the optical (Table 8 and Figures 2-4), and from M4.5 to M6 in the NIR (Table 7). For an accreting object, such changes are most easily ascribed to changes in veiling, i.e., changes in the excess continuum

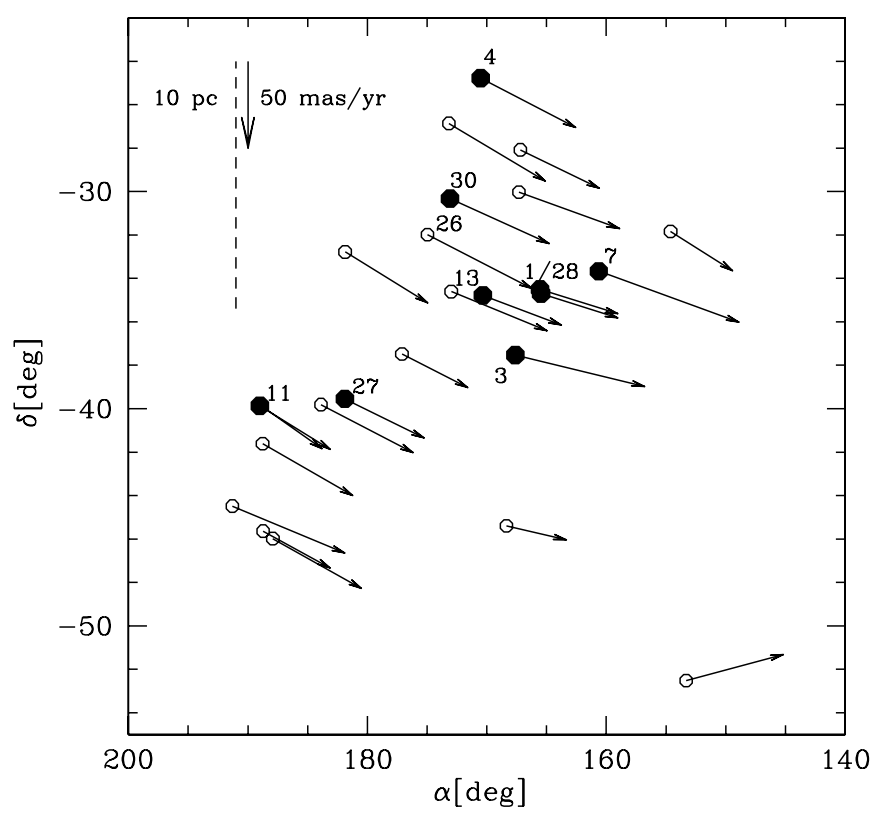

Figure 12. Spatial locations of all TWA members with disks (filled circles) and without disks (empty circles) based on mid-IR excesses, with the exception of TWA 30, which has no mid-IR data available but shows spectroscopic signs of a disk. Members with a disk are also labeled by their TWA designation. Note that most members with a disk reside in the north-west quadrant of the TWA. The short-dashed line marks a distance of $10 \mathrm{pc}$ at the mean distance of the TWA members $(53 \pm 3 \mathrm{pc})$, and the arrow indicates the scale length for the proper motions of members.

emission due to accretion. In this view, the earliest spectral type corresponds to maximum accretion, and the latest spectral type to minimum accretion.

This interpretation is supported by the behavior of the photospheric lines of Li I $\lambda 6708$ and K I $\lambda 7699$ (point 5). Both show variable absorption: the smallest absorption EWs (indeed, KI goes into emission) are seen in 2009, when the optical spectral type is earliest, while the largest absorption widths occur in 2009 March and May, when the optical types are the latest. For photospheric absorption, this cannot be due simply to changes in the stellar continuum (e.g., due to suppression of the continuum by dust occlusion), since the lines and continuum would be equally affected without changing the EW. Instead, it points to a significant enhancement of accretion during this period which partially fills in (veils) the Li I absorption through excess continuum emission and also produces excess line emission in $\mathrm{K}$. The observed correlation between decreasing absorption strength (i.e., enhanced accretion) and earlier spectral type then 
confirms that the changes in the underlying optical spectral type are due to veiling effects. By Occam's razor, the similar variations in the NIR spectral type may then also be attributed to accretion-induced veiling effects. Note that if this veiling interpretation is correct, then the true spectral type of the star is likely to be $\sim$ M5.25-M6, i.e., the latest and thus least veilingaffected types we see in the optical and NIR. Nevertheless, given the additional complexity introduced by reddening variability (see the next subsection), we have simply adopted M5 \pm 1 which covers the full range seen in the data; a more accurate classification will have to await time-resolved high-resolution spectroscopy.

\subsubsection{Observed Relationship Between Reddening, Accretion, and Wind Signatures}

Moreover, we also see the least reddening $A_{V}$ in both the optical and the NIR, when the underlying spectral type is earliest, and intermediate (in the optical) to highest (in the NIR) $A_{V}$ when the inferred spectral type is the latest (point 4). If the spectral type changes are due to variable accretion and if the extinction is due to dust in the line of sight, as we argue, then this implies that the dust is mostly anti-correlated with accretion. A physical interpretation of this is supplied further below.

P Cygni profiles (point 6) are clear signatures of cool outflowing winds along the line of sight moving toward the observer (e.g., Edwards et al. 2003, and references therein). Their sporadic appearance in the $\mathrm{Na}$ I D lines, and once weakly in $\mathrm{H} \alpha$ (2009 March, when the Na I D P Cygni profiles are also strongest), implies that the wind from TWA 30 intersects our line of sight only occasionally. Moreover, the P Cygni profiles are strongest in 2009 March and May, when the apparent optical spectral type is latest, M5.25, and absorption in photospheric Li I and KI is greatest, i.e., by our above arguments, when the accretion is weakest. Conversely, the P Cygni profiles are weakest/absent in 2009 January, when the optical spectral type is earliest, M4, and the $\mathrm{Li} \mathrm{I}$ and $\mathrm{K}$ I absorption is weakest-i.e., when accretion is strongest. It is noteworthy that the $\mathrm{Na}$ I D doublet evinces the strongest, somewhat redshifted emission in the same 2009 January spectra. This cannot be explained simply by the absence of blueshifted P Cygni emission, but instead again indicates enhanced accretion when the P Cygni wind signatures are weakest.

There are two possible explanations for this behavior. The first is that the P Cygni profiles are always present at similar strength, but completely filled in by accretion-induced veiling when accretion is strongest. The other is that there is a real weakening of the wind signatures when accretion is strongest. While some veiling of absorption profiles clearly occurs during enhanced accretion (e.g., in Li I), we suggest that the second effect - a real weakening of the wind-is likely to be present as well, due to the following physically motivated argument.

\subsubsection{A Physical Interpretation: An Inclined Stellar Magnetosphere Interacting with the Disk Inner Edge}

We have noted earlier that the extinction/reddening signatures, caused by dust in the line of sight, are anti-correlated with accretion, while now we find that the P Cygni wind signatures show the same behavior. Both can be explained simultaneously by the interaction of a tilted stellar magnetosphere interacting with the inner edge of the accretion disk. In this case, accretion is energetically preferred along one half of the inner-edge circumference (where the field lines bend inward toward the star) while outflow is preferred along the other half (where the field lines bend outward toward the disk). As the star rotates, an observer viewing the disk edge-on along either the top or bottom disk surface would preferentially see outflow and accretion $180^{\circ}$ out of phase in time. This is precisely what is seen, for instance, in the cTTS SU Aur (e.g., Johns \& Basri 1995), and would explain the alternating P Cygni wind signatures and enhanced accretion we observe in TWA 30. Furthermore, the interaction of an inclined magnetosphere is expected to create a disk warp on theoretical grounds (Terquem \& Papaloizou 2000), which would explain the variable reddening observed in TWA 30. In the case of AA Tau, where such a warp has also been invoked (Bouvier et al. 2003), it appears associated with the accretion funnel flow, i.e., is correlated instead of anti-correlated with the accretion as we observed for TWA 30. However, Terquem \& Papaloizou (2000) show that the specific sense of the warp-i.e., whether the warp vertical extension is parallel or anti-parallel to that of the accretion funnel flow or wind-depends on particular assumptions about the nature of the field at the disk inner edge. Hence, a disk warp associated with the wind base is possible as well. In summary, this single physical situation can explain the variable extinction, variable wind signatures, variable accretion, and apparent anti-correlation of the two former with the latter. On the other hand, if only increased veiling were responsible for the disappearance of the wind-related P Cygni profiles during periods of enhanced accretion, we have no obvious reason for the reddening to be anti-correlated with accretion. We therefore propose that there is in fact a real change in the wind signatures. This must be checked through future high-resolution, time-resolved optical spectroscopy, wherein the wavelength-dependent veiling can be characterized in detail.

\subsubsection{Further Evidence for an Edge-on Geometry, and Implications for Accreting Gas}

We further see that the P Cygni profiles are strong in $\mathrm{Na}$ I D but weak in $\mathrm{H} \alpha$, the $\mathrm{Na}$ I $\mathrm{D}$ absorption is only moderately blueshifted from the stellar rest-frame (mean $\sim 25 \mathrm{~km} \mathrm{~s}^{-1}$; Figure 7 ), and the $\mathrm{H} \alpha$ emission varies by a factor of 1.8 (following the same trend as other accretion signatures - such as veiling and line emission in $\mathrm{K}$ I and Na I D_of being enhanced in 2009 January compared to 2009 March and May). All these are consistent with the edgeon hypothesis. In this geometry, our line of sight intersects the cool dense base of the flow where the resonance lines of $\mathrm{Na}$ I and $\mathrm{K}$ I are easily excited but not $\mathrm{H} \alpha$, so we are more sensitive to changes in accretion and outflow in the former lines than in the latter; this is also where a magneto-centrifugal wind has the smallest velocity (and we see an even smaller component of it in our edge-on line of sight; Edwards 2007; Kwan et al. 2007), yielding only a small shift in RV in wind signatures relative to the stellar rest frame.

In this regard, we also note that in many edge-on systems redshifted, inverse P Cygni absorption is seen superimposed on the $\mathrm{H} \alpha$ emission, arising from infalling gas seen against the hotter accretion shock on the stellar surface. No such feature is apparent in our $\mathrm{H} \alpha$ profiles. This may be because the accreting gas is optically thick, completely obscuring the shock region, as supported by the lack of detectable emission in He I at 4471, 6678 , or $7065 \AA$, as well as very weak narrow emission in He I $5876 \AA$ (the strongest line in the series). In cTTS, He I appears to be produced in both the accretion flow, giving rise to a broad component, and in the shock, yielding a narrow component. The absence of a broad He I component in our data is consistent with an edge-on geometry, since this is a high-temperature line that would not be excited at the cool base of the flow. The absence or 
extreme weakness of a He I narrow component in the presence of other accretion signatures, further suggests that the shock region is obscured by optically thick accreting gas.

\subsubsection{Evidence for Time Variability in the FELs, and a Physical Interpretation}

The scenario sketched so far is consistent with spatial variations alone: the changes in the accretion/outflow/extinction signatures, due to inner-disk interactions with a tilted stellar magnetosphere, occur as the accretion flow, outflow and disk warp rotate into and out of our line of sight. As such, these signatures should be periodic on the scale of the rotation period of the disk inner edge. In cTTS, this is usually on the order of days. Our observations are very poorly sampled at such short periods, and thus remain consistent with this picture of spatial changes alone. We note that the very strong increase in NIR reddening in 2009 May over a period of only 6 days hints at changes on the timescales expected from rotational effects. This will need to be verified by future finely time-sampled monitoring.

However, spatial variability alone cannot be the whole story. Considering the FELs in TWA 30 (point 7), we note first that the EWs of nearly all of these are weakest in the 2009 March and May optical data and strongest in the 2009 January optical data. This could be explained if the stellar photosphere were severely occulted in 2009 January relative to 2009 March and May; i.e., if the FELs were seen in 2009 January against a much dimmer continuum. However, this appears in contradiction to our argument above, where the optical data indicate that it is 2009 January when the $A_{V}$ is least (indicating minimum dust reddening and occlusion) and 2009 March and May when the $A_{V}$ is intermediate to highest (indicating maximum reddening/ occlusion effects). Moreover, the EWs of different FELs change by different amounts, while they should all change by the same proportion if it is the stellar continuum that is being suppressed. The FELs are also expected to arise on scales of at least a few AU from the star, and thus cannot themselves be expected to be affected by any occultation mechanisms operating near the star (e.g., a disk warp).

The only alternative is that the FELs are intrinsically time variant. FEL time variations may arise as the jet encounters inhomogeneities in its path, or represent temporal changes in the jet launching. If the latter occurs in TWA 30, then the strengthening of the P Cygni profiles in 2009 March and May may indicate an actual increase in the outflow rate at these epochs instead of just the outflow rotating into our line of sight. Such a scenario is not inconsistent with the FELs being weakest in these epochs. An FEL can only arise when the critical density of that particular species is reached, at a distance of tens of AUs for cTTS to perhaps a few AU for brown dwarfs (Whelan et al. 2009). At the very least, there must be a time lag between the observation (via P Cygni profiles) of a strengthening in the wind at its base, and the corresponding increase in the FELs a few AU away, representing the jet travel time. Assuming a fiducial jet velocity of $\sim 300 \mathrm{~km} \mathrm{~s}^{-1}$ and a distance of $\sim 5$ AU to the critical density region, yields a time lag of $\sim 30$ days.

Our interpretation of the FEL variations is then simply that at some point in the past there was a strengthening in the outflow, which led (after some time lag) to the brightening of the FELs in the 2009 January spectra. By the time of the 2009 March and May spectroscopy, the FELs have faded back to their normal levels. The fact that these epochs coincide with an increase in accretion and wind signatures near the star, respectively, would simply be coincidental. This is a plausible scenario if changes in the latter signatures occur episodically (and maybe periodically) on timescales of days. We note further that, relative to the 2008 November data, the FELs in the 2009 January data are stronger: in [O I] $\lambda 6300$ by $49 \%$, in [S II] $\lambda 6731$ by $165 \%$, and in [N II] $\lambda 6583$ by $63 \%$ (see Table 8 ). This may be understood by noting that the requisite critical electron density is highest for [O I], smaller for [S II], and least for [N II]. Thus, for a jet whose streamlines diverge (i.e., density decreases) with increasing distance, the $\left[\mathrm{O}_{\mathrm{I}}\right]$ emission region lies closest to the star, [S II] appears further out, and [N II] lies furthest away, as found earlier by Hirth et al. (1997) for cTTS. We thus posit that the past outflow enhancement has nearly reached the [O I] critical region by 2008 November but has yet to arrive at the [S II] and [N II] locations; by 2009 January the [O I] FELs have plateaued and the outflow enhancement has arrived in the [S II] region, brightening this line considerably, but has not quite reached [N II] yet; and at last, by 2009 March and May, the enhancement has passed all these regions, and all the FELs have faded away again.

To clarify this model, multiple observations are needed: finely time-sampled photometry to measure the periodicity of the disk rotation/outflow, mid-IR photometry to detect the disk, midIR spectroscopy to detect the presence of silicates and infer grain sizes, high-resolution broadband imaging to resolve the disk, high-resolution narrowband imaging to resolve the outflows, polarimetry to investigate the dust-disk geometry, spectroastrometry to spatially map the outflow, and submillimeter observations to measure the disk mass. As one of the few very low mass stars/brown dwarfs for which many of these observations are possible, TWA 30 could prove to be an invaluable case study for accretion and outflow processes at the low-mass end.

\section{CONCLUSION}

We have identified a new and unusual member of the TWA, TWA 30, which has emission lines of [O I], [O II], [O III], [S II], and $\mathrm{Mg}$ I] near the stellar rest velocity, indicating it is powering an outflow. The temporal changes in reddening, absorption/emission EWs, and line profiles, particularly for $\mathrm{Na}$ I D, suggests a stellar magnetospheric axis inclined with respect to a disk that is viewed nearly edge-on. The presence of forbidden line emission [O III] and $\mathrm{Mg}$ I] in the spectra of TWA 30 marks the first time these lines have been seen in a young star not residing in a molecular cloud and are rarely present even amongst cTTS in star formation regions. Both the optical and NIR spectral types appear to vary in accordance with the reddening from M4-M5.25 and from M4.5-M6, respectively, so we have adopted a spectral type of M5 \pm 1 . We suggest that the earlier spectral types are seen at periods of high accretion and include continuum excess emission. The spectral type of TWA 30 therefore might be as late as M5.25-M6. From evolutionary models, we estimate an age of $7 \pm 3 \mathrm{Myr}$ and a mass of $0.12 \pm$ $0.04 M_{\odot}$. The close proximity of TWA $30,42 \pm 2$ pc, makes it an excellent target for follow-up studies to spatially resolve the outflow and circumstellar disk. With the inclusion of TWA 30, we have updated the disk census of the TWA, finding that $35_{-8}^{+11} \%$ of observed TWA members still retain their circumstellar disks, a higher ratio than previous estimates.

We thank our anonymous referee whose comments improved the quality of this paper. We thank George Herbig, Mike Cushing, Nathan Smith, Silvia Alencar, Silvie Cabrit, Bo Reipurth, Klaus Hodapp, Brendan Bowler, Kevin Covey, George Wallerstein, and Suzanne Hawley for useful discussions. 
We also thank our telescope operators at Magellan: Mauricio Martinez, Hernán Nuñez, and Ricardo Covarrubias, and at IRTF: Paul Sears, Bill Golisch, and Dave Griep. D.L.L. thanks Dave Sanders and George Herbig for financial support. This research has benefitted from the $\mathrm{M}, \mathrm{L}$, and $\mathrm{T}$ dwarf compendium housed at DwarfArchives.org and maintained by Chris Gelino, J. Davy Kirkpatrick, and Adam Burgasser. This research has made use of the Atomic Line List v2.04 maintained at http://www.pa.uky.edu/ peter/atomic/; the SIMBAD database and VizieR catalog access tool, operated at CDS, Strasbourg, France; and the facilities of the Canadian Astronomy Data Centre operated by the National Research Council of Canada with the support of the Canadian Space Agency. This paper uses data from the IRTF Spectral Library (http://irtfweb.ifa.hawaii.edu/ spex/IRTF_Spectral_Library/ index.html) maintained by John Rayner, Michael Cushing, and William Vacca. This publication makes use of data products from the Two Micron All Sky Survey, which is a joint project of the University of Massachusetts and the Infrared Processing and Analysis Center/California Institute of Technology, funded by the National Aeronautics and Space Administration and the National Science Foundation. This research has made use of the NASA/IPAC Infrared Science Archive, which is operated by the Jet Propulsion Laboratory, California Institute of Technology, under contract with the National Aeronautics and Space Administration. As some spectroscopic follow-up data were obtained from the summit of Mauna Kea, the authors wish to recognize and acknowledge the very significant and cultural role and reverence that this mountaintop has always had with the indigenous Hawaiian community. We are most fortunate to have the opportunity to conduct observations there.

\section{REFERENCES}

Adams, F. C., Lada, C. J., \& Shu, F. H. 1987, ApJ, 312, 788

Alexander, R. D., \& Armitage, P. J. 2006, ApJ, 639, L83

Appenzeller, I., \& Mundt, R. 1989, AA\&R, 1, 291

Baraffe, I., Chabrier, G., Allard, F., \& Hauschildt, P. H. 1998, A\&A, 337, 403

Barrado y Navascués, D. 2006, A\&A, 459, 511

Bernstein, R., Shectman, S. A., Gunnels, S. M., Mochnacki, S., \& Athey, A. E. 2003, Proc. SPIE, 4841, 1694

Bessell, M. S., \& Brett, J. M. 1988, PASP, 100, 1134

Biller, B. A., \& Close, L. M. 2007, ApJ, 669, L41

Bochanski, J. J., West, A. A., Hawley, S. L., \& Covey, K. R. 2007, AJ, 133, 531 Bochanski, J. J., et al. 2009, PASP, 121, 1409

Böhm, K.-H. 1995, Ap\&SS, 233, 11

Bourke, T. L., Crapsi, A., Myers, P. C., Evans, N. J., II, Wilner, D. J., Huard,

T. L., Jørgensen, J. K., \& Young, C. H. 2005, ApJ, 633, L129

Bouvier, J., et al. 1999, A\&A, 349, 619

Bouvier, J., et al. 2003, A\&A, 409, 169

Bouvier, J., et al. 2007, A\&A, 463, 1017

Brandeker, A., Jayawardhana, R., \& Najita, J. 2003, AJ, 126, 2009

Calvet, N., Muzerolle, J., Briceño, C., Hernández, J., Hartmann, L., Saucedo, J. L., \& Gordon, K. D. 2004, AJ, 128, 1294

Cardelli, J. A., Clayton, G. C., \& Mathis, J. S. 1989, ApJ, 345, 245

Carpenter, J. M. 2001, AJ, 121, 2851

Chabrier, G., \& Baraffe, I. 2000, ARA\&A, 38, 337

Chambers, J. E. 2004, Earth Planet. Sci. Lett., 223, 241

Chauvin, G., Lagrange, A.-M., Dumas, C., Zuckerman, B., Mouillet, D., Song, I., Beuzit, J.-L., \& Lowrance, P. 2004, A\&A, 425, L29

Cruz, K. L., Kirkpatrick, J. D., \& Burgasser, A. J. 2009, AJ, 137, 3345

Cushing, M. C., Vacca, W. D., \& Rayner, J. T. 2004, PASP, 116, 362

Cutri, R. M., et al. 2003, VizieR Online Data Catalog, 2246, 0

de la Reza, R., Torres, C. A. O., Quast, G., Castilho, B. V., \& Vieira, G. L. 1989, ApJ, 343, L61

Delfosse, X., Forveille, T., Perrier, C., \& Mayor, M. 1998, A\&A, 331, 581

Ducourant, C., Teixeira, R., Chauvin, G., Daigne, G., Le Campion, J.-F., Song, I., \& Zuckerman, B. 2008, A\&A, 477, L1

Edwards, S. 2007, in IAU Symp. 243, Star-Disk Interaction in Young Stars, ed. J. Bouvier \& I. Appenzeller (Cambridge: Cambridge Univ. Press), 171
Edwards, S., Fischer, W., Kwan, J., Hillenbrand, L., \& Dupree, A. K. 2003, ApJ, 599, L41

Enoch, M. L., Evans, N. J., II, Sargent, A. I., Glenn, J., Rosolowsky, E., \& Myers, P. 2008, ApJ, 684, 1240

Epchtein, N., et al. 1997, Messenger, 87, 27

Ercolano, B., Drake, J. J., Raymond, J. C., \& Clarke, C. C. 2008, ApJ, 688, 398

Fedele, D., van den Ancker, M. E., Henning, T., Jayawardhana, R., \& Oliveira, J. M. 2009, arXiv:0911.3320

Fernández, M., \& Comerón, F. 2001, A\&A, 380, 264

Finch, C. T., Zacharias, N., \& Wycoff, G. L. 2010, arXiv:1002.0556

Fleming, T. A., Schmitt, J. H. M. M., \& Giampapa, M. S. 1995, ApJ, 450, 401

Gizis, J. E. 2002, ApJ, 575, 484

Gizis, J. E., Jao, W.-C., Subasavage, J. P., \& Henry, T. J. 2007, ApJ, 669, L45

Golimowski, D. A., et al. 2004, AJ, 127, 3516

Gregorio-Hetem, J., Lepine, J. R. D., Quast, G. R., Torres, C. A. O., \& de La Reza, R. 1992, AJ, 103, 549

Güdel, M., et al. 2007, A\&A, 468, 353

Hambly, N. C., Davenhall, A. C., Irwin, M. J., \& MacGillivray, H. T. 2001a, MNRAS, 326, 1315

Hambly, N. C., Irwin, M. J., \& MacGillivray, H. T. 2001b, MNRAS, 326, 1295

Hambly, N. C., et al. 2001c, MNRAS, 326, 1279

Hartigan, P. 1989, ApJ, 339, 987

Hartigan, P., Edwards, S., \& Ghandour, L. 1995, ApJ, 452, 736

Hartmann, L. 1998, Accretion Processes in Star Formation (Cambridge Astrophys. Ser; Cambridge: Cambridge Univ. Press)

Henize, K. G. 1976, ApJS, 30, 491

Herbig, G. H. 1978, in Problems of Physics and Evolution of the Universe, ed. L.V. Mirzoyan (Yerevan: Publishing House of the Armenian Academy of Sciences), 171

Herbig, G. H. 1990, ApJ, 360, 639

Herczeg, G. J., Cruz, K. L., \& Hillenbrand, L. A. 2009, ApJ, 696, 1589

Herczeg, G. J., \& Hillenbrand, L. A. 2008, ApJ, 681, 594

Hirth, G. A., Mundt, R., \& Solf, J. 1997, A\&AS, 126, 437

Jayawardhana, R., Hartmann, L., Fazio, G., Fisher, R. S., Telesco, C. M., \& Piña, R. K. 1999, ApJ, 521, L129

Johns, C. M., \& Basri, G. 1995, ApJ, 449, 341

Johnson, D. R. H., \& Soderblom, D. R. 1987, AJ, 93, 864

Kamiński, T., Schmidt, M., Tylenda, R., Konacki, M., \& Gromadzki, M. 2009, ApJS, 182, 33

Kastner, J. H., Zuckerman, B., Weintraub, D. A., \& Forveille, T. 1997, Science, 277, 67

Kenyon, S. J., \& Hartmann, L. 1995, ApJS, 101, 117

Kirkpatrick, J. D., Barman, T. S., Burgasser, A. J., McGovern, M. R., McLean, I. S., Tinney, C. G., \& Lowrance, P. J. 2006, ApJ, 639, 1120

Kirkpatrick, J. D., Henry, T. J., \& McCarthy, D. W., Jr. 1991, ApJS, 77, 417

Kwan, J., Edwards, S., \& Fischer, W. 2007, ApJ, 657, 897

Lawson, W. A., \& Crause, L. A. 2005, MNRAS, 357, 1399

Lawson, W. A., Crause, L. A., Mamajek, E. E., \& Feigelson, E. D. 2001, MNRAS, 321, 57

Leggett, S. K. 1992, ApJS, 82, 351

Looper, D. L., Burgasser, A. J., Kirkpatrick, J. D., \& Swift, B. J. 2007, ApJ, 669, L97

Low, F. J., Smith, P. S., Werner, M., Chen, C., Krause, V., Jura, M., \& Hines, D. C. 2005, ApJ, 631, 1170

Luhman, K. L. 1999, ApJ, 525, 466

Luhman, K. L. 2004, ApJ, 616, 1033

Luhman, K. L., \& Muench, A. A. 2008, ApJ, 684, 654

Luhman, K. L., Stauffer, J. R., Muench, A. A., Rieke, G. H., Lada, E. A., Bouvier, J., \& Lada, C. J. 2003, ApJ, 593, 1093

Luhman, K. L., \& Steeghs, D. 2004, ApJ, 609, 917

Mamajek, E. E. 2005, ApJ, 634, 1385

Mamajek, E. E., Lawson, W. A., \& Feigelson, E. D. 1999, ApJ, 516, L77

Marshall, J. L., et al. 2008, Proc. SPIE, 7014, 169

Martín, E. L., Basri, G., \& Zapatero Osorio, M. R. 1999, AJ, 118, 1005

Masciadri, E., \& Raga, A. C. 2004, ApJ, 615, 850

Meyer, M. R., Calvet, N., \& Hillenbrand, L. A. 1997, AJ, 114, 288

Mohanty, S., \& Basri, G. 2003, ApJ, 583, 451

Mohanty, S., Jayawardhana, R., \& Barrado y Navascués, D. 2003, ApJ, 593, L109

Mohanty, S., Jayawardhana, R., \& Basri, G. 2005, ApJ, 626, 498

Monet, D., et al. 1998, VizieR Online Data Catalog, 1252, 0

Monet, D. G., et al. 2003, AJ, 125, 984

Morrow, A. L., et al. 2008, ApJ, 676, L143

Murray, C. A. 1989, A\&A, 218, 325

Muzerolle, J., Hillenbrand, L., Calvet, N., Briceño, C., \& Hartmann, L. 2003, ApJ, 592, 266 
Muzerolle, J., Hillenbrand, L., Calvet, N., Hartmann, L., \& Briceño, C. 2001, in ASP Conf. Ser. 244, Young Stars Near Earth: Progress and Prospects, ed. R. Jayawardhana \& T. Greene (San Francisco, CA: ASP), 245

Natta, A., Testi, L., Muzerolle, J., Randich, S., Comerón, F., \& Persi, P. 2004, A\&A, 424, 603

Nelson, L. A., Rappaport, S., \& Chiang, E. 1993, ApJ, 413, 364

O’Donnell, J. E. 1994, ApJ, 422, 158

Phan-Bao, N., et al. 2008, ApJ, 689, L141

Plavchan, P., Werner, M. W., Chen, C. H., Stapelfeldt, K. R., Su, K. Y. L., Stauffer, J. R., \& Song, I. 2009, ApJ, 698, 1068

Raga, A. C., Böhm, K.-H., \& Cantó, J. 1996, RevMexAA, 32, 161

Rayner, J. T., Cushing, M. C., \& Vacca, W. D. 2009, ApJS, 185, 289

Rayner, J. T., Toomey, D. W., Onaka, P. M., Denault, A. J., Stahlberger, W. E., Vacca, W. D., Cushing, M. C., \& Wang, S. 2003, PASP, 115, 362

Reiners, A. 2009, ApJ, 702, L119

Riaz, B., \& Gizis, J. E. 2008, ApJ, 681, 1584

Riaz, B., Gizis, J. E., \& Hmiel, A. 2006, ApJ, 639, L79

Rieke, G. H., \& Lebofsky, M. J. 1985, ApJ, 288, 618

Rucinski, S. M., \& Krautter, J. 1983, A\&A, 121, 217

Scholz, R.-D., McCaughrean, M. J., Zinnecker, H., \& Lodieu, N. 2005, A\&A, 430, L49

Schwartz, R. D. 1983, ARA\&A, 21, 209

Shu, F. H., Adams, F. C., \& Lizano, S. 1987, ARA\&A, 25, 23

Skrutskie, M. F., et al. 2006, AJ, 131, 1163

Soderblom, D. R., et al. 1998, ApJ, 498, 385

Song, I., Zuckerman, B., \& Bessell, M. S. 2003, ApJ, 599, 342

Sterzik, M. F., Alcalá, J. M., Covino, E., \& Petr, M. G. 1999, A\&A, 346, L41

Sterzik, M. F., Pascucci, I., Apai, D., van der Bliek, N., \& Dullemond, C. P. 2004, A\&A, 427, 245
Tachihara, K., Neuhäuser, R., \& Fukui, Y. 2009, PASJ, 61, 585

Terquem, C., \& Papaloizou, J. C. B. 2000, A\&A, 360, 1031

Torres, C. A. O., Quast, G. R., Melo, C. H. F., \& Sterzik, M. F. 2008, in Handbook of Star Forming Regions: Vol. II, The Southern Sky, ed. B. Reipurth (San Francisco, CA: ASP), 757

Vacca, W. D., Cushing, M. C., \& Rayner, J. T. 2003, PASP, 115, 389

van der Bliek, N. S., et al. 2004, Proc. SPIE, 5492, 1582

van Leeuwen, F. 2007, A\&A, 474, 653

Voges, W., et al. 1999, A\&A, 349, 389

Voges, W., et al. 2000, IAU Circ., 7432, 3

Webb, R. A., Zuckerman, B., Platais, I., Patience, J., White, R. J., Schwartz, M. J., \& McCarthy, C. 1999, ApJ, 512, L63

Weingartner, J. C., \& Draine, B. T. 2001, ApJ, 548, 296

Weintraub, D. A., Saumon, D., Kastner, J. H., \& Forveille, T. 2000, ApJ, 530 867

West, A. A., \& Basri, G. 2009, ApJ, 693, 1283

West, A. A., Hawley, S. L., Bochanski, J. J., Covey, K. R., Reid, I. N., Dhital, S., Hilton, E. J., \& Masuda, M. 2008, AJ, 135, 785

Whelan, E. T., Ray, T. P., \& Bacciotti, F. 2009, ApJ, 691, L106

Whelan, E. T., Ray, T. P., Bacciotti, F., Natta, A., Testi, L., \& Randich, S. 2005, Nature, 435, 652

Whelan, E. T., Ray, T. P., Randich, S., Bacciotti, F., Jayawardhana, R., Testi, L., Natta, A., \& Mohanty, S. 2007, ApJ, 659, L45

White, R. J., \& Basri, G. 2003, ApJ, 582, 1109

White, R. J., \& Ghez, A. M. 2001, ApJ, 556, 265

Wyatt, M. C., Dent, W. R. F., \& Greaves, J. S. 2003, MNRAS, 342, 876

Zuckerman, B., \& Song, I. 2004, ARA\&A, 42, 685

Zuckerman, B., Webb, R. A., Schwartz, M., \& Becklin, E. E. 2001, ApJ, 549, L233 